\title{
Disruption of CEP290 microtubule/membrane-binding domains causes retinal degeneration
}

\author{
Theodore G. Drivas, ${ }^{1}$ Erika L.F. Holzbaur, ${ }^{2}$ and Jean Bennett ${ }^{1}$ \\ ${ }^{1}$ F.M. Kirby Center for Molecular Ophthalmology and ${ }^{2}$ Department of Physiology and Pennsylvania Muscle Institute, \\ Perelman School of Medicine, University of Pennsylvania, Philadelphia, Pennsylvania, USA.
}

\begin{abstract}
Mutations in the gene centrosomal protein $290 \mathrm{kDa}($ CEP290) cause an array of debilitating and phenotypically distinct human diseases, ranging from the devastating blinding disease Leber congenital amaurosis (LCA) to Senior-Løken syndrome, Joubert syndrome, and the lethal Meckel-Gruber syndrome. Despite its critical role in biology and disease, very little is known about CEP290's function. Here, we have identified 4 functional domains of the protein. We found that CEP290 directly binds to cellular membranes through an $\mathrm{N}$-terminal domain that includes a highly conserved amphipathic helix motif and to microtubules through a domain located within its myosin-tail homology domain. Furthermore, CEP290 activity was regulated by 2 autoinhibitory domains within its $\mathrm{N}$ and $\mathrm{C}$ termini, both of which were found to play critical roles in regulating ciliogenesis. Disruption of the microtubule-binding domain in a mouse model of LCA was sufficient to induce significant deficits in cilium formation, which led to retinal degeneration. These data implicate CEP290 as an integral structural and regulatory component of the cilium and provide insight into the pathological mechanisms of LCA and related ciliopathies. Further, these data illustrate that disruption of particular CEP290 functional domains may lead to particular disease phenotypes and suggest innovative strategies for therapeutic intervention.
\end{abstract}

\section{Introduction}

Upon exit from the cell cycle and entry into $\mathrm{G}_{0}$, nearly every human cell sequesters its centrosome in a structure known as the primary cilium (1). Until recently, the functional role of the primary cilium, a single antenna-like projection of the apical membrane, had not been well studied or appreciated. It is now clear that this organelle has important sensory roles affecting multiple cellular processes (2-4) and that defects in primary cilium formation and function are responsible for a variety of human diseases and developmental disorders, collectively termed ciliopathies (5).

While the ciliopathies are diverse in both phenotype and etiology, specific genes, including centrosomal protein $290 \mathrm{kDa}$ (CEP290), have been implicated as having causative roles in multiple ciliumassociated disorders (6). CEP290 mutations have been described in up to $20 \%$ of cases of the devastating inherited blinding disease Leber congenital amaurosis $(7,8)$ and in numerous cases of other more debilitating ciliopathies, such as Joubert syndrome (9-11), Senior-Løken syndrome (12), and Meckel-Gruber syndrome (13). How the many identified mutations in CEP290 contribute to these diverse pathologies remains unknown, and the protein's normal biological role has not been well characterized.

Throughout the cell cycle, CEP290 localizes to both the mother and daughter centrioles $(9,14)$, where it is maintained in an inhibited state by the protein CP110 (15). During $\mathrm{G}_{0}$, the kinase Ttbk2 mediates the removal and degradation of CP110 from CEP290 at the mother centriole $(16,17)$, leading to ciliogenesis, the formation of the primary cilium. Ciliogenesis is driven by the highly regulated and specific transport of ciliary proteins into the ciliary

Conflict of interest: Jean Bennett is a cofounder of GenSight Biologics and serves on the scientific advisory board of Avalanche Biotechnologies.

Citation for this article: J Clin Invest. 2013;123(10):4525-4539. doi:10.1172/JCI69448. compartment through a process known as intraflagellar transport (IFT) (18). Numerous studies have demonstrated that CEP290's function is critical for this process - in CEP290 knockdown experiments,proteins that would normally localize to the cilium fail to do so $(14,19)$ and cilium formation is disrupted $(20,21)$.

Once the cilium is formed, CEP290 is found at the ciliary transition zone, the region of the organelle just proximal to the microtubule axoneme. Elegant structural work in the Chlamydomonas model system has localized CEP290 to the ciliary Y-links (19), dense, proteinaceous structures within the transition zone that closely couple the ciliary membrane to its microtubule core (22). The Y-links are thought to form a barrier to passive diffusion between the cilium and the cytosol (23) and possibly act as a scaffold for the cellular machinery responsible for IFT (24). CEP290 likely plays an important role in the organization of this diffusion barrier and "ciliary pore" through the recruitment of a number of interacting partners, such as CC2D2A (25), NPHP5 (26), and Rab8 (20), all of which are also involved in cilium function and disease. However, despite our expanding knowledge of CEP290 interacting partners and localization, the precise role CEP290 plays in ciliogenesis, IFT, and as a component of the Y-links is still unclear. No functional domains for the protein have been identified, and how mutations in particular regions of the gene affect protein function and cause disease remains completely unknown.

In this paper, we present evidence that CEP290 directly binds to cellular membranes through a highly conserved region in its $\mathrm{N}$ terminus and to microtubules through a domain located near its C terminus. CEP290 activity was found to be regulated by autoinhibitory domains located within its $\mathrm{N}$ and $\mathrm{C}$ termini, both of which were found to play a critical role in regulating ciliogenesis. Furthermore, we show that the microtubule-binding domain we identified is completely disrupted in the rd16 mouse Leber congen- 
A

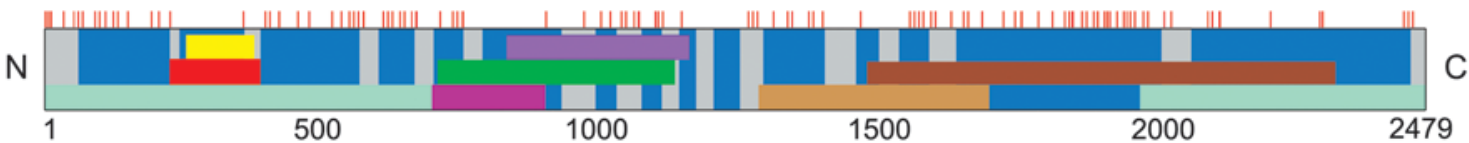

Homo/heterodimerization domains

CC2D2A binding domain

\section{$\square$ Coiled-coil}

SMC homology domain

CP110 binding domain

PCM-1 binding domain

NPHP5 binding domain

B FL
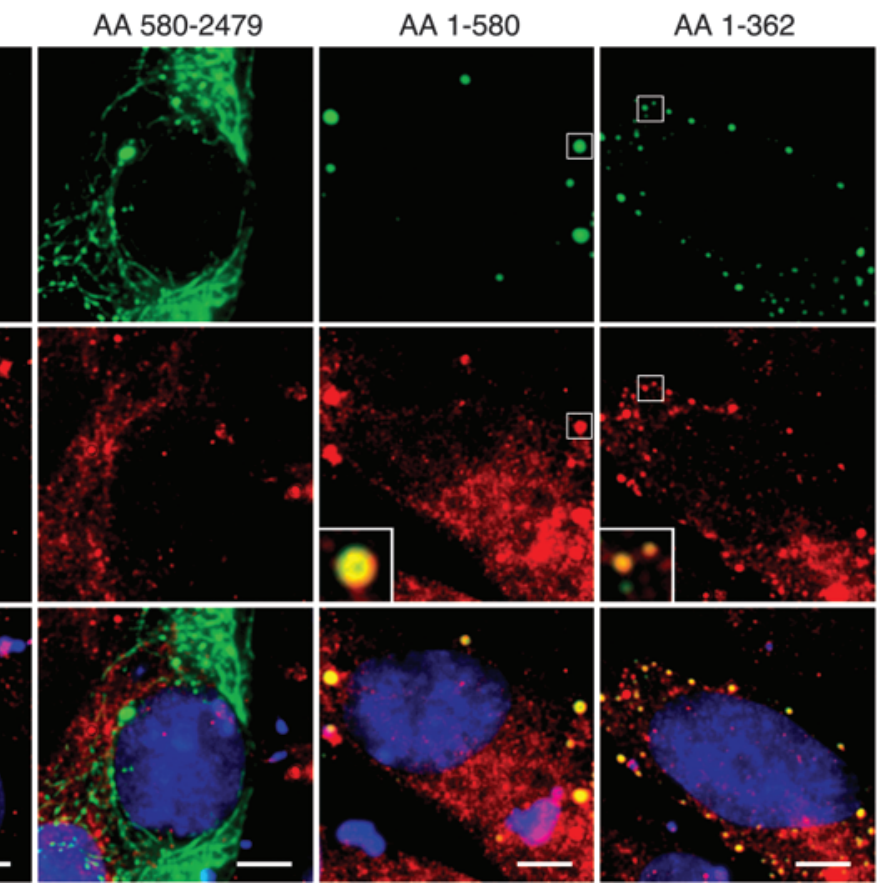

Myosin-tail homology domain
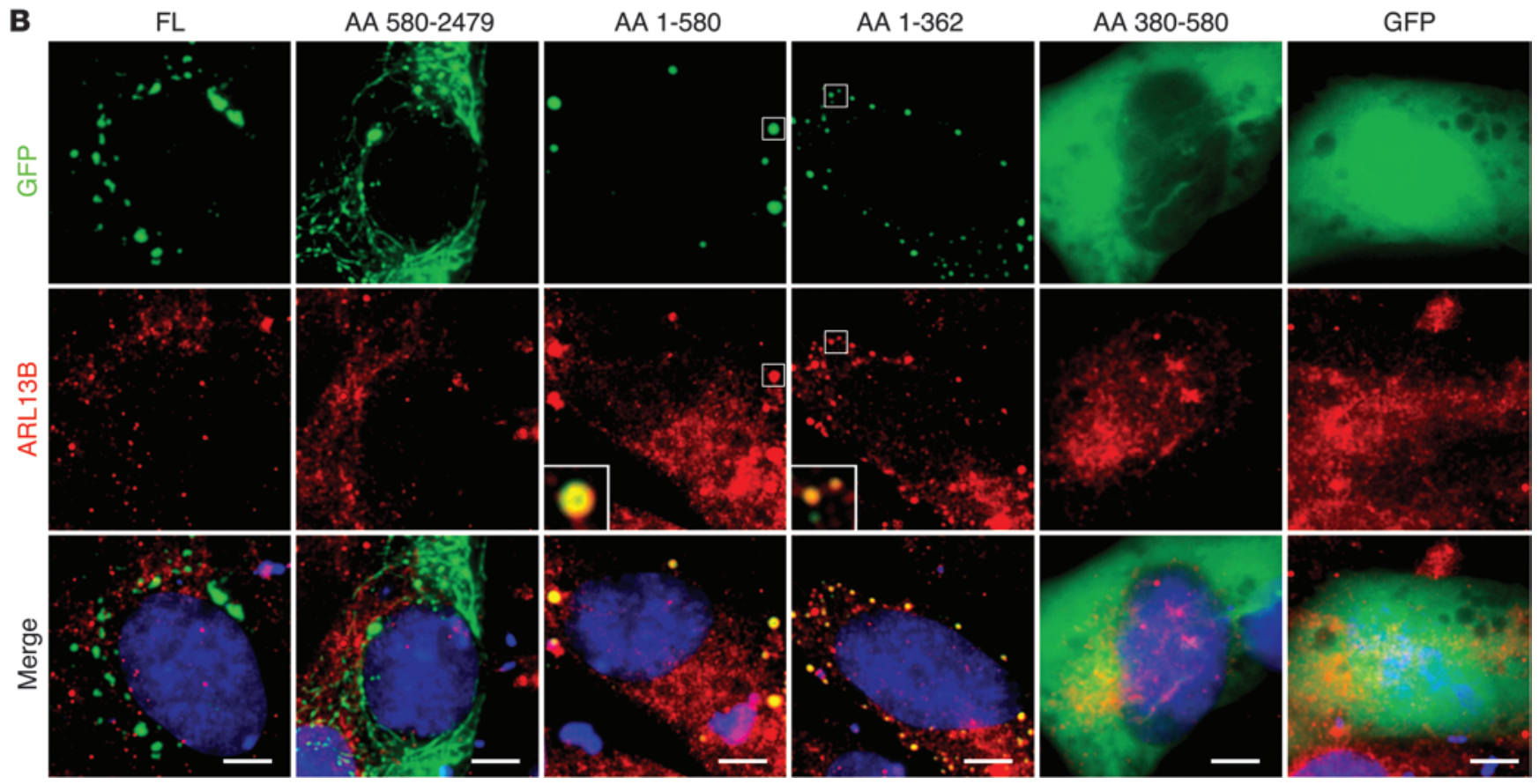

Figure 1

CEP290 aa 1-362 mediate punctuate localization. (A) Scale representation of human CEP290. Known human CEP290 mutations are noted by red tick marks. The indicated domains and mutations are adapted from ref. 30. (B) Fluorescence microscopy images showing the localization pattern of GFP fusions of full-length (FL) and truncated CEP290 constructs in cycling hTERT-RPE1 cells. Cells were stained with an antibody against ARL13B and with DAPI. Scale bars: $5 \mu \mathrm{m}$. Insets show $\times 3$ magnified views of areas (in boxes) of colocalization between CEP290 truncations and ARL13B. Pearson correlation coefficient for colocalization between ARL13B and CEP290 aa 1-580 is 0.53 and for CEP290 aa 1-362 is 0.65.

ital amaurosis (LCA) model (14), resulting in significant deficits in cilium formation leading to retinal degeneration. These findings implicate CEP290 both as a key structural component of the ciliary Y-links and as a terminal regulator in the pathway leading to ciliogenesis. Our study also provides what we believe is the first evidence of a mechanistic and pathological basis for CEP290-related LCA and related ciliopathies and suggests strategies for therapeutic intervention.

\section{Results}

Mutations in the CEP290 gene have been implicated in a variety of human diseases (27), but their effects on protein function have not yet been characterized. Mutations clustering in particular regions of the gene might be indicative of important functional domains, but no mutational hot spots or functional domains have been identified to date (Figure 1A and ref. 28). To better understand the role CEP290 plays in cilium function, ciliogenesis, and human disease, we performed a structure-function analysis using a panel of truncation constructs spanning the full length of CEP290 to identify and define domains of novel functionality.
CEP290 associates with ARL13B-positive cellular membranes via its $N$ terminus. The first 2 CEP290 truncations we tested displayed distinct localization patterns. When overexpressed as GFP fusion proteins, the N-terminal fragment of CEP290 (spanning aa 1-580) showed an exclusively punctate localization pattern, while the C-terminal fragment of CEP290 (from aa 580 to the end of the protein, aa 2479) showed a striking fibrillar localization (Figure 1B). Both of these patterns were occasionally observed in cells overexpressing the full-length CEP290 construct, but not with the same frequency as in cells expressing the truncations (data not shown). We generated 2 additional truncations of the $\mathrm{N}$-terminal region of the protein to better define the domain responsible for the punctate localization. The truncation spanning CEP290 aa 1-362 showed a punctate pattern similar to that observed for the complete N-terminal fragment, while the truncation spanning aa $380-580$ was present only diffusely throughout the cytoplasm (Figure 1B). Further truncation of the protein was not effective in resolving the domain responsible for punctate localization beyond aa 1-362, implying either that this is the minimum region needed for punctate staining or that further truncation substantially interferes with protein function. 
A

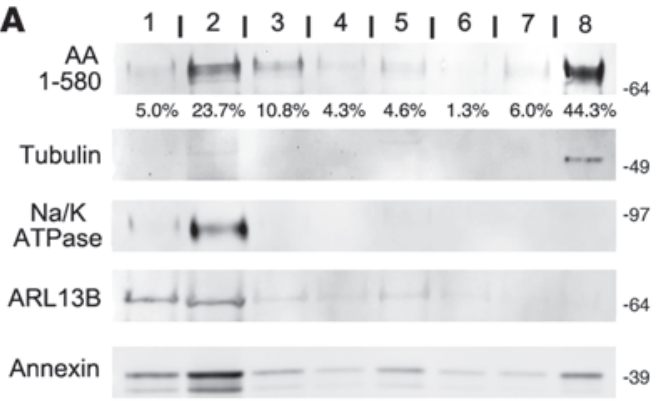

B

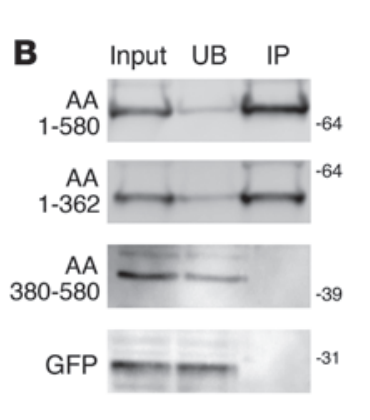

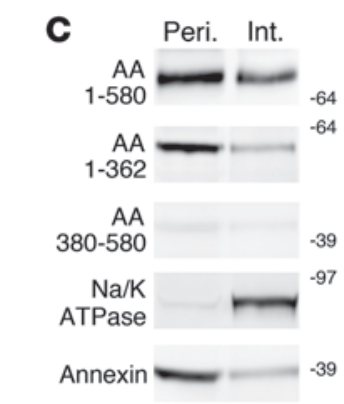

D
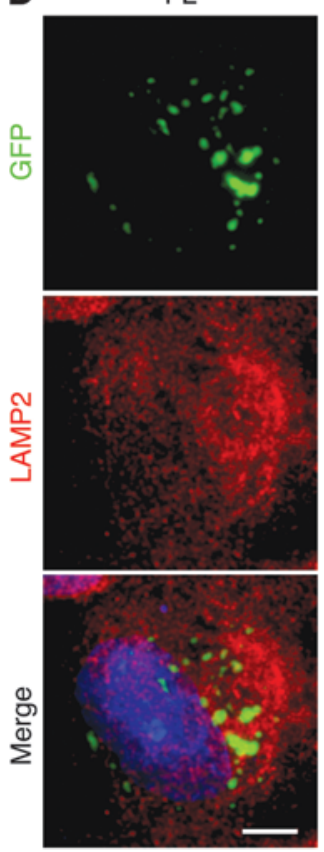

$50 \%$ Membrane Associated

\section{$80 \%$}

Soluble

\section{AA $580-2479$}
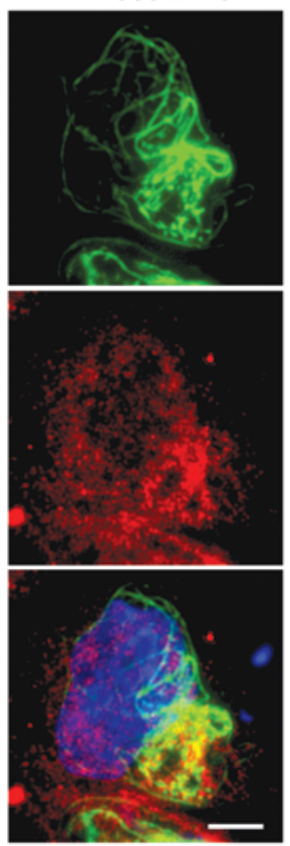

AA $1-580$
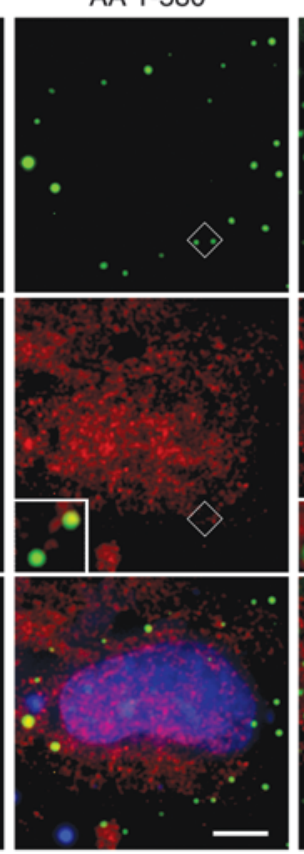

AA 1-362
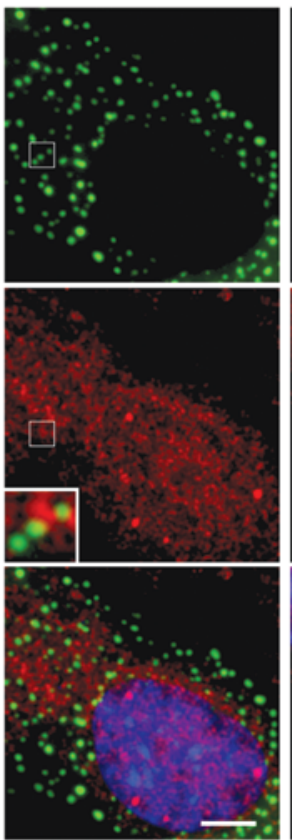

AA $380-580$
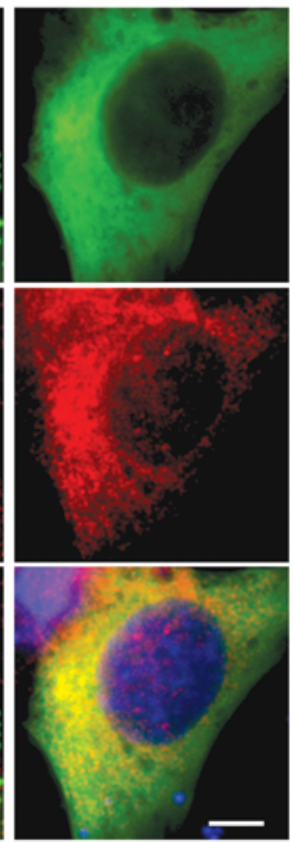

GFP
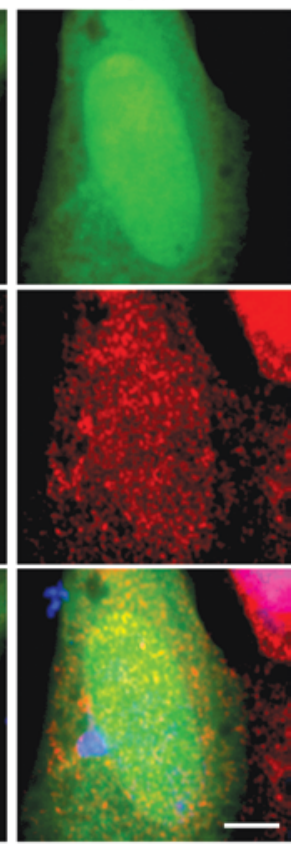

Figure 2

CEP290 aa 1-362 mediate peripheral membrane association. (A) Equal amounts of each of 5 fractions, beginning from the top (row 1) and ending at the bottom (row 8) of the sucrose gradient of a membrane flotation assay performed on hTERT-RPE1 cells overexpressing CEP290 aa 1-580. Sucrose percentages and the percentage of CEP290 aa 1-590 found in each fraction are indicated. Blots were probed for GFP to detect CEP290 aa 1-580 and for the indicated controls. (B) ARL13B-positive membranes immunoprecipitated from postnuclear supernatants of hTERTRPE1 cells expressing the indicated GFP-fused CEP290 truncations or GFP alone were probed with an anti-GFP antibody. $15 \%$ of the input fraction, $15 \%$ of the unbound fraction (UB), and the entire immunoprecipitated fraction (IP) were loaded. (C) The peripheral (Peri.) and integral (Int.) membrane protein fractions of hTERT-RPE1 cells expressing the indicated GFP-fused CEP290 truncations were isolated and probed for GFP and for the indicated controls. (D) Fluorescence microscopy images showing the localization pattern of GFP-fused full-length and truncated CEP290 constructs in hTERT-RPE1 cells stained for LAMP2 and with DAPI (blue). Scale bars: $5 \mu \mathrm{m}$. Insets show $\times 3$ magnified views of areas (in boxes) illustrating lack of colocalization between CEP290 truncations and LAMP2.

The CEP290-positive puncta observed by microscopy were found to display robust costaining with ARL13B (Figure 1B), a membrane protein important in the trafficking of vesicles to the primary cilium (29-31), suggesting that these puncta might be cilium-destined cellular vesicles. Detergent-free postnuclear supernatants of cells expressing our CEP290 truncations were prepared, and ARL13B-positive membranes were magnetically immunoprecipitated and analyzed by Western blotting. Both CEP290 aa 1-580 and CEP290 aa 1-362 were significantly enriched in the ARL13B membrane immunoprecipitate, while neither CEP290 aa 380-580 nor GFP alone were found in significant quantities (Figure 2B). Thus, CEP290 aa 1-362 were found to be necessary and sufficient to mediate CEP290 localization to ARL13B-positive membranes. In only very few cases did the CEP290-positive puncta exhibit any costaining with LAMP2, a marker of the lysosomal compartment (Figure 2D), suggesting that CEP290 membrane localization was not an artifact of protein overexpression.

To confirm that the puncta observed by microscopy were truly membranous organelles, we performed a series of membrane coflotation assays on cells expressing CEP290 aa 1-580. Roughly 
A

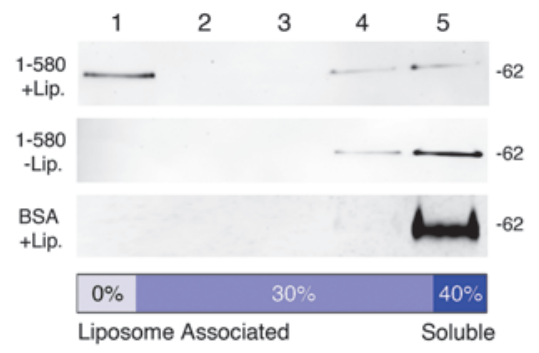

B

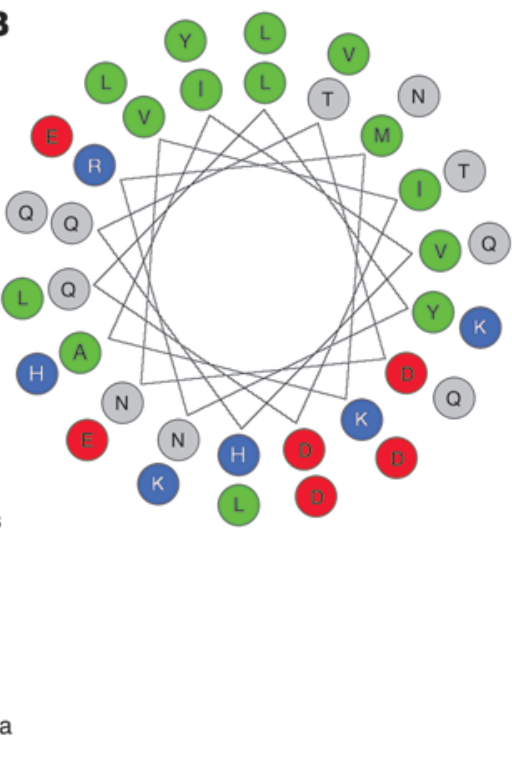

\section{Figure 3}

CEP290 aa 1-362 mediates direct membrane binding. (A) Liposome coflotation assays performed on purified CEP290 aa 1-580, both with and without liposomes, and with BSA as a control. Equal amounts of each of 5 fractions, beginning from the top (row 1 ) and ending at the bottom (row 5) of the sucrose gradient were analyzed. Sucrose percentages and the expected protein composition of each fraction are indicated. (B) Helical wheel projection of CEP290 aa 257-292. Red circles represent negatively charged amino acids, blue circles represent positively charged amino acids, grey circles represent polar, uncharged amino acids, and green circles represent nonpolar amino acids. Adapted from the Helical Wheel Projection applet (see Methods). (C) Multiple sequence alignment of CEP290's predicted amphipathic helix. Amino acids are color coded using the same scheme as in F. Adapted from GeneDoc 2.7.000 (see Methods).

$40 \%$ of total CEP290 aa 1-580 was found to cofloat in the membrane-associated fractions (Figure 2A, fractions 1-3), indicating that a substantial portion of the protein was, in fact, associated with cellular membranes. ARL13B, the $\mathrm{Na} / \mathrm{K}$ ATPase, and annexin A2 were all found to similarly cofloat in the membrane-associated fractions, while tubulin was found almost exclusively in the soluble fraction (Figure 2A).

The dual distribution of CEP290 aa 1-580 within both the membrane-associated and soluble fractions along with the absence of a signal peptide from CEP290's aa sequence was suggestive of peripheral, rather than integral, membrane association $(32,33)$. To confirm this, membrane fractions of cells transfected with our CEP290 truncations were prepared and peripheral membrane proteins were eluted from the membrane with a high $\mathrm{pH}$ buffer. The remaining integral membrane proteins were subsequently solubilized with detergent. For both CEP290 aa 1-580 and aa $1-362$, the majority of each truncation was found in the peripheral membrane protein fraction (Figure $2 \mathrm{C}$ ). This same pattern was observed for the peripheral membrane protein annexin A2 (34), while the majority of the $\mathrm{Na} / \mathrm{K}$ ATPase, an integral membrane protein, was found in the integral membrane protein fraction (Figure 2C). CEP290 aa 380-580, on the other hand, was not found in significant amounts in either fraction. Taken together, these data indicate that CEP290 aa 1-362 are necessary and sufficient for robust peripheral membrane association.

CEP290's capacity for membrane association is increased by truncation of its C terminus. To further investigate CEP290's membrane association, we performed a series of subcellular fractionation experiments on cells expressing each of our CEP290 truncations. The CEP290 truncation spanning aa 580-2479 that produced a fibrillar localization pattern by microscopy was found almost exclusively in the cytoskeletal fraction, while truncations lacking this region were completely absent from the cytoskeletal fraction (Supplemental Figure 1A; supplemental material available online with this article; doi:10.1172/JCI69448DS1). On the other hand, truncations that included CEP290 aa 1-362 were again found to be significantly present in the membrane fraction when compared with either GFP alone or our fractionation controls (Supplemental Figure 1A). Roughly 30\% each of both CEP290 truncations aa 1-580 and aa 1-362 were found to be associated with cellular membranes, while roughly $10 \%$ of full-length CEP290 was also found in the membrane fraction (Supplemental Figure 1B). This distribution agrees with what was observed by fluorescence microscopy - specifically, that full-length CEP290 occasionally displayed vesicular localization, but to a lesser extent than CEP290 truncations lacking the $\mathrm{C}$ terminus of the protein but containing aa $1-362$.

CEP290 directly binds membranes in vitro and contains a bighly conserved membrane-binding amphipathic $\alpha$-helix motif. To determine whether CEP290's membrane association was mediated by a direct or indirect interaction, we performed a series of liposome coflotation assays on purified recombinant CEP290 aa 1-580 (Supplemental Figure 2A). CEP290 aa 1-580 associated with liposomes robustly, with a majority of the protein found in the liposome-associated fraction (Figure 3A). Flotation of the truncation occurred only in the presence of liposomes, and liposome coflotation was not observed for a control protein, BSA (Figure 3A). The ability of this region of CEP290 to directly bind liposomes suggests that the observed association between the N terminus of CEP290 and cellular membranes is mediated by a direct interaction. 


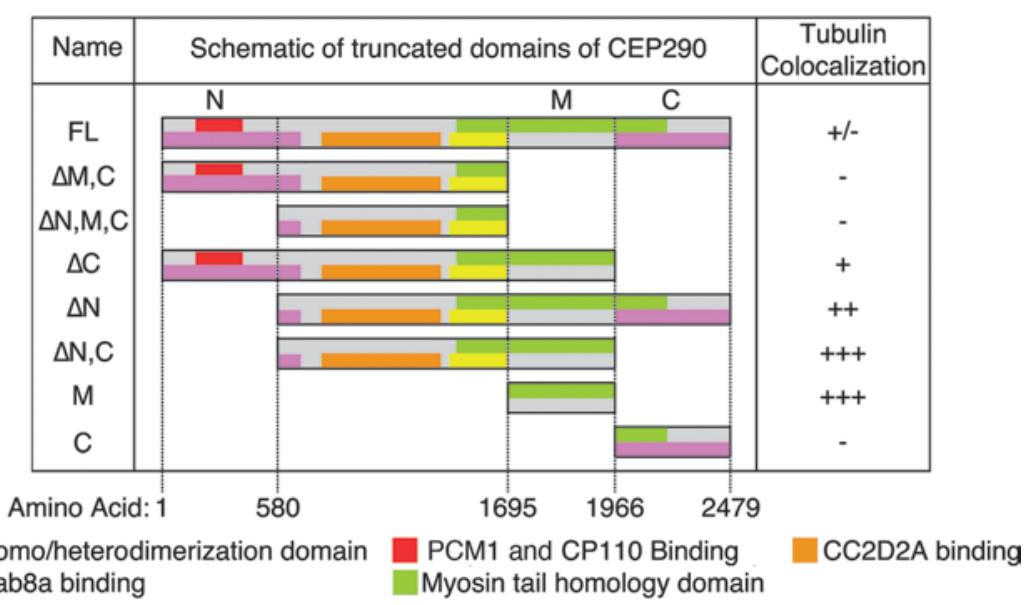

Projecting CEP290 aa 1-362 onto an $\alpha$-helical wheel, a segment from aa 257 to 292 was predicted to form a canonical amphipathic $\alpha$-helix (Figure 3B). Such helices have been shown to be criti$\mathrm{cal}$ in mediating robust interactions between peripheral membrane proteins and various cellular membranes (35). Comparing this stretch of the protein across a variety of species, we found the amphipathic helix motif to be very highly conserved. Where there was divergence in the amino acid sequence, there was usually conservation of polarity and charge between the divergent residues (Figure 3C). Taken together, these data suggest that the highly conserved amphipathic helix located within the membrane binding region of CEP290 may be mediating CEP290's novel membrane-binding function.

CEP290 a 1695-1966 mediate colocalization with microtubules. CEP290 aa 580-2479 appeared both by microscopy and subcellular fractionation to be associated with the cytoskeleton (Figure 1B, Figure 2D, and Supplemental Figure 1A). To further investigate this phenomenon, we constructed a library of additional CEP290 truncations to thoroughly interrogate CEP290's cytoskeletal association (Figure 4). Overexpression of these truncations as GFP fusions revealed that those truncations containing CEP290 aa 1695-1966, referred to as region M, displayed a fibrillar localization pattern similar to that which was observed for CEP290 aa 580-2479 while truncations lacking CEP290 region $M$ never display any fibrillar localization (Figure 5, A and B). These fibrils were noted to colocalize with the microtubule network implicating CEP290 region M as necessary and sufficient for microtubule colocalization.

The degree to which different CEP290 truncations displayed a fibrillar localization pattern was found to be dependent upon which regions of the protein were included in the truncation. The full-length CEP290 construct was noted to produce a fibrillar localization pattern in only about $10 \%$ of transfected cells (Figure 5B). Truncations lacking either the $\mathrm{N}$ or $\mathrm{C}$ terminus of CEP290, on the other hand, were found to display a fibrillar localization in roughly $20 \%$ and $60 \%$ of transfected cells, respectively. The truncation lacking both termini was found to display a fibrillar localization pattern in nearly $80 \%$ of transfected cells. These data indicated that the $\mathrm{N}$ and $\mathrm{C}$ termini of the protein have an inhibitory or regulatory effect on CEP290's microtubule binding ability.

It is interesting to note that all of our truncations displayed colocalization with pericentrin, a marker of the centriole (the nor-

\section{Figure 4}

Schematic of CEP290 truncations. Scale representations of the CEP290 truncations tested in Figures $5-7$. Included is a summary of the extent of colocalization with tubulin, rated on a scale ranging from negative (-) to highly positive (+++). mal site of CEP290 localization) (Figure 5A, insets). Homotypic interactions between endogenous CEP290, present at the centrioles, and our truncations (through CEP290's homo/heterodimerization domains; ref. 26) might explain the observed centriolar localization of a number of our truncations. However, those truncations lacking both homo/heterodimerization domains are apparently still capable of localizing to the centriole, implying that multiple regions throughout CEP290 are capable of affecting centriolar localization.

CEP290 microtubule association results in microtubule acetylation and bundling. In cells transfected with CEP290 constructs containing region $M$, there was a dramatic increase in the intensity of acetylated $\alpha$-tubulin staining, with bundles of acetylated microtubules looping throughout the cells (Figure 6A). These increases were not seen for truncations lacking region $M$. The degree to which different CEP290 truncations increased the acetylation and bundling of microtubules was dependent upon which regions of the protein were included in the truncation. Full-length CEP290 and CEP290 truncations lacking either the $\mathrm{N}$ or $\mathrm{C}$ terminus increased microtubule acetylation and bundling in nearly $40 \%$ of transfected cells, while CEP290 truncations lacking both the $\mathrm{N}$ and C termini of the protein increased microtubule acetylation and bundling in nearly $75 \%$ of cells (Figure 6B). Less than $15 \%$ of cells transfected with truncations lacking region $M$ were noted to have any change in microtubule acetylation or bundling.

CEP290 directly binds microtubules in vitro. A series of microtubule cosedimentation assays performed on our CEP290 truncation constructs revealed that truncations containing region $\mathrm{M}$ were capable of significant microtubule association in vitro (Figure 7, A and B). Again, the degree to which different CEP290 truncations associated with microtubules was found to be dependent on the inclusion of the $\mathrm{N}$ and $\mathrm{C}$ termini. Less than $50 \%$ of the full-length CEP290 construct and CEP290 constructs lacking either terminus were found to associate with microtubules, while nearly 100\% of CEP290 constructs lacking both termini associated with microtubules (Figure 7B).

To test whether CEP290 region M's microtubules association was mediated by direct microtubule binding, we recombinantly expressed and purified CEP290 region M (Supplemental Figure 2B) and subjected this protein to a series of microtubule cosedimentation assays using increasing concentrations of microtubules (Figure 7, $\mathrm{C}$ and $\mathrm{D})$. Region $\mathrm{M}$ was found to directly and robustly bind to 
A

A $\quad \mathrm{FL}$

$\mathrm{FL} \quad \Delta \mathrm{M}, \mathrm{C}$

$\triangle \mathrm{N}, \mathrm{M}, \mathrm{C}$

$\Delta C$

$\Delta \mathrm{N}$

$\Delta \mathrm{N}, \mathrm{C}$
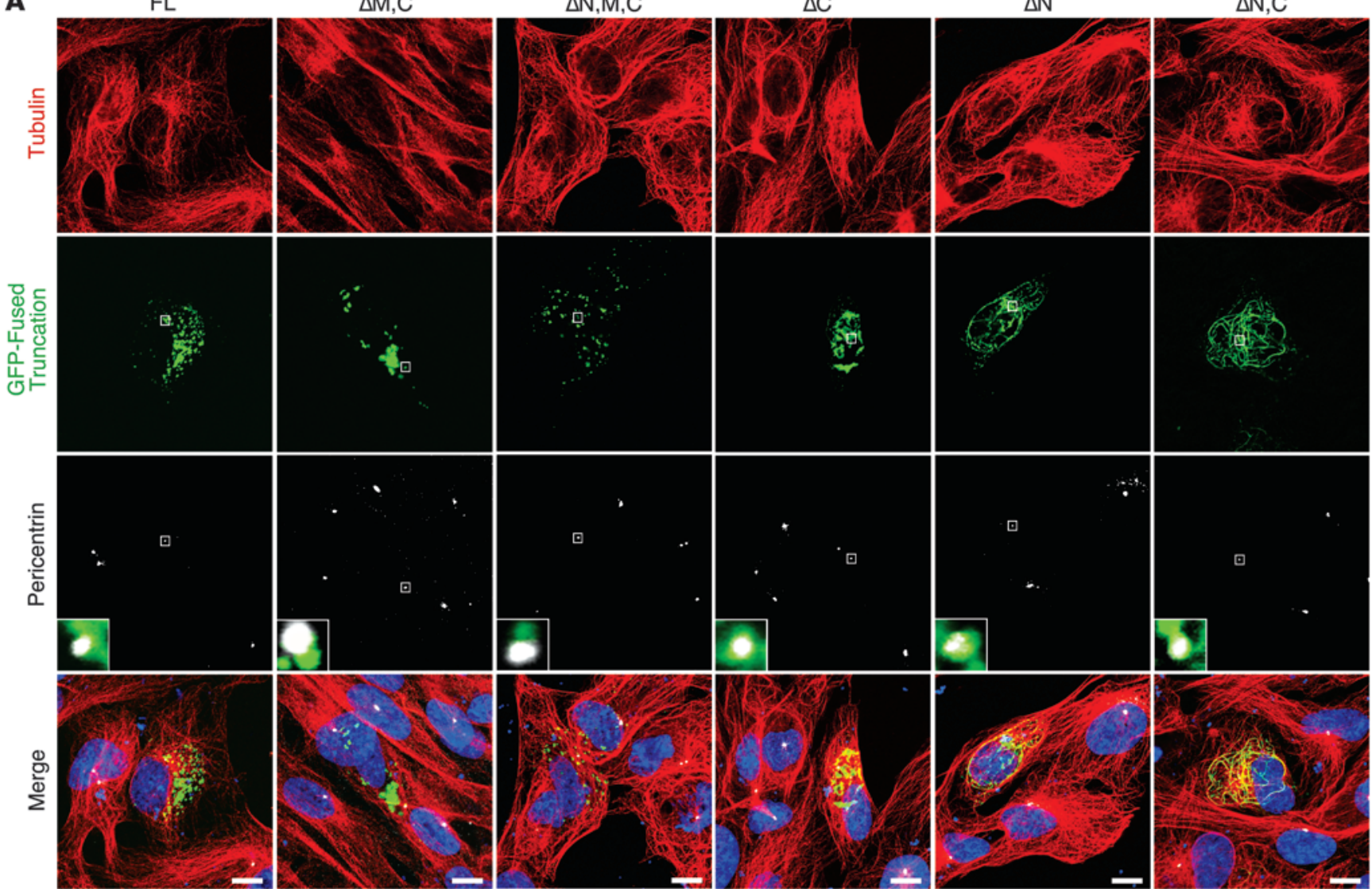

M

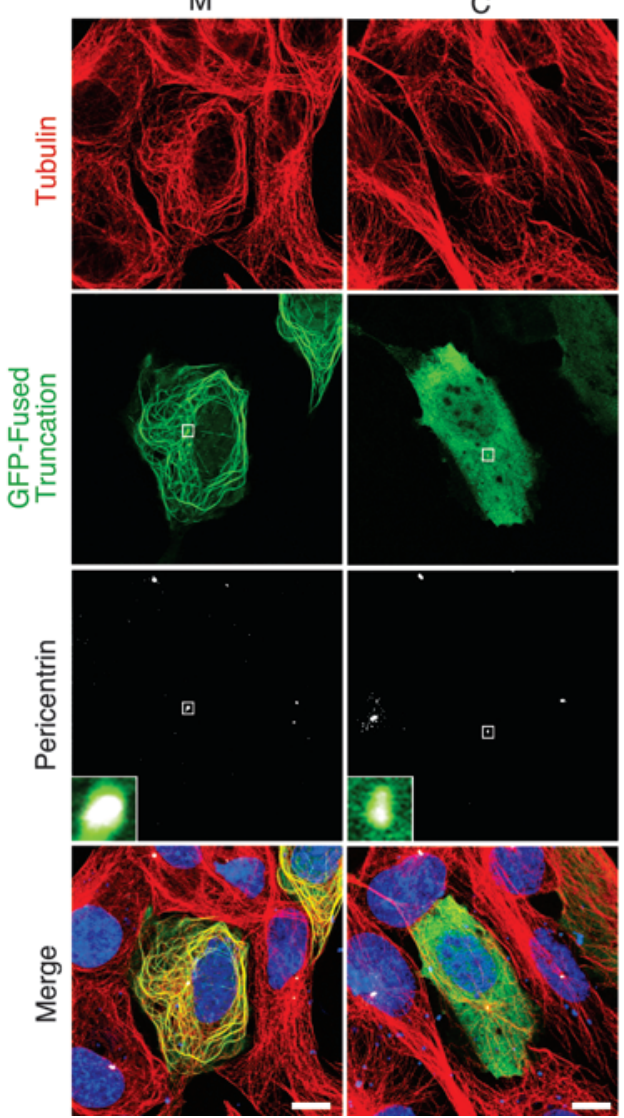

B

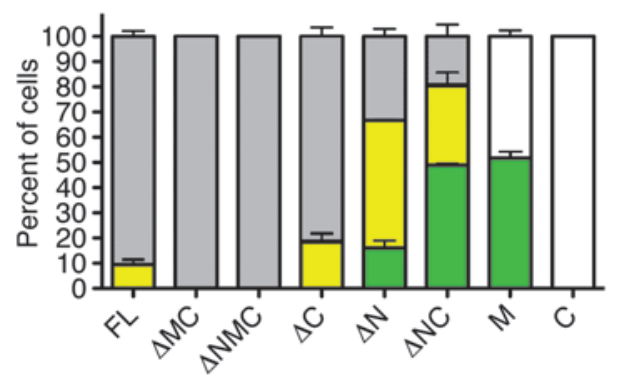

Figure 5

CEP290 region M mediates microtubule colocalization. (A) Fluorescence confocal microscopy images showing the localization pattern of GFP-fused full-length and truncated CEP290 constructs expressed in hTERT-RPE1 cells. Samples were stained for $\alpha$-tubulin and pericentrin and with DAPI (blue). Scale bars: $10 \mu \mathrm{m}$. Insets show $\times 10$ magnified views of areas of colocalization (in boxes) between CEP290 truncations and pericentrin. (B) The percentage of transfected hTERT-RPE1 cells in which GFP-fused truncations displayed a fibrillar localization pattern. At least 100 cells were counted per experiment. Data are presented as mean $\pm \mathrm{SD}, n=3$. 

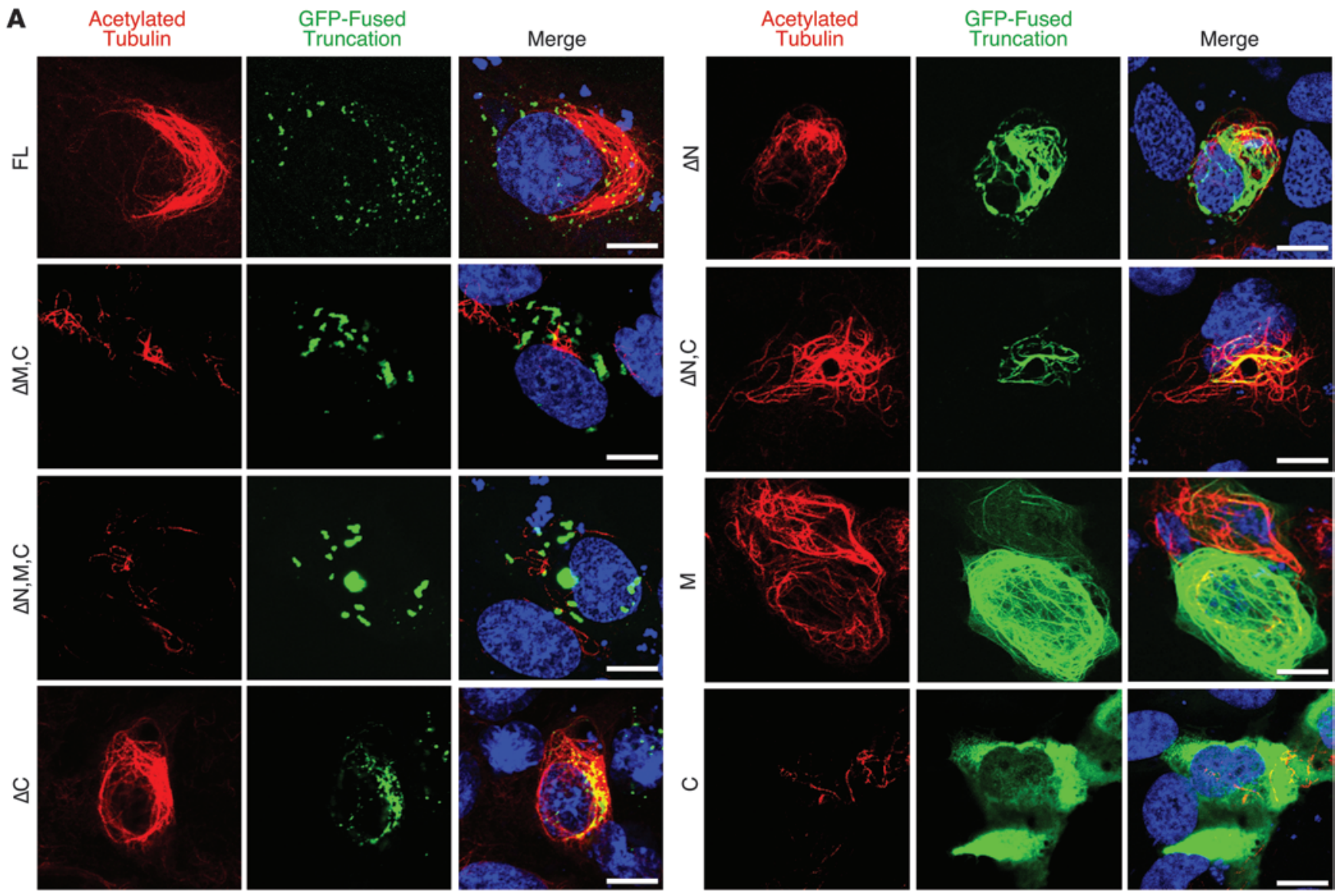

B

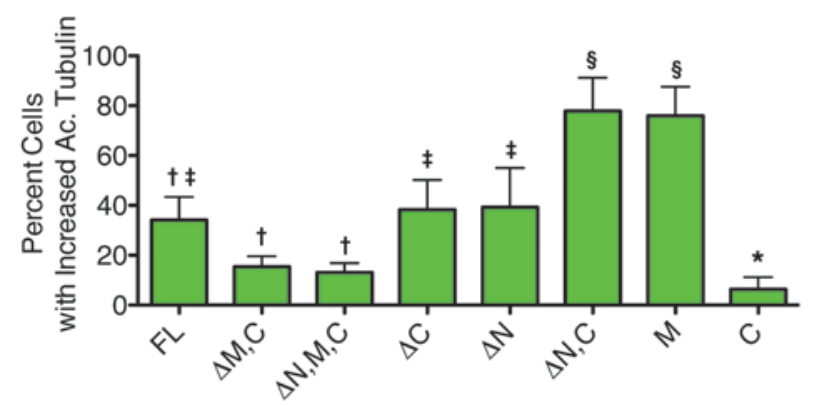

Figure 6

CEP290 region M mediates tubulin acetylation. (A) Representative confocal fluorescence microscopy images of GFP-fused full-length and truncated CEP290 constructs expressed in hTERT-RPE1 cells stained for acetylated $\alpha$-tubulin and with DAPI (blue). Scale bars: $10 \mu \mathrm{m}$. (B) Percentage of 293T cells showing an increase in the intensity of acetylated $\alpha$-tubulin staining following transfection with CEP290 constructs. 100 transfected cells were counted per experiment. Data are presented as mean $\pm \mathrm{SD}, n=5$. Symbols indicate significance as follows: ${ }^{\star} P<0.05$; † $P<0.05 ; ¥ P<0.05 ; \S P<0.05$.

microtubules in a concentration-dependent manner (Figure 7D). The calculated $\mathrm{K}_{\mathrm{d}}$ of this interaction was found to be approximately $100 \mathrm{nM}$, an affinity comparable to those of other microtubule binding proteins $(36,37)$.

The $N$ and $C$ termini of CEP290 cooperate to inbibit protein function and regulate ciliogenesis. The observation that the $\mathrm{N}$ and $\mathrm{C}$ termini of CEP290 appeared to act in inhibiting the membrane- and microtubule-binding activity of the protein (Figure 4, Figure 5B, Figure $6 \mathrm{~B}$, Figure 7B, and Supplemental Figure 1B) suggested that these regions might be regulatory domains mediating the autoinhibi- tion of the protein. To test this hypothesis, we transduced hTERTRPE1 cells with lentiviral vectors encoding either the $\mathrm{N}$ or $\mathrm{C}$ terminus of the protein and observed the cells for deficits in primary cilium formation. To our surprise, we found that overexpression of either of these regulatory regions resulted not in deficiencies in primary cilium formation, but instead in significant increases in the percentage of cells forming primary cilia, with more than twice as many cells forming primary cilia than those cells treated with a control vector (Figure 8, A and B). The length of cilia formed by cells overexpressing either regulatory region was also signifi- 
A
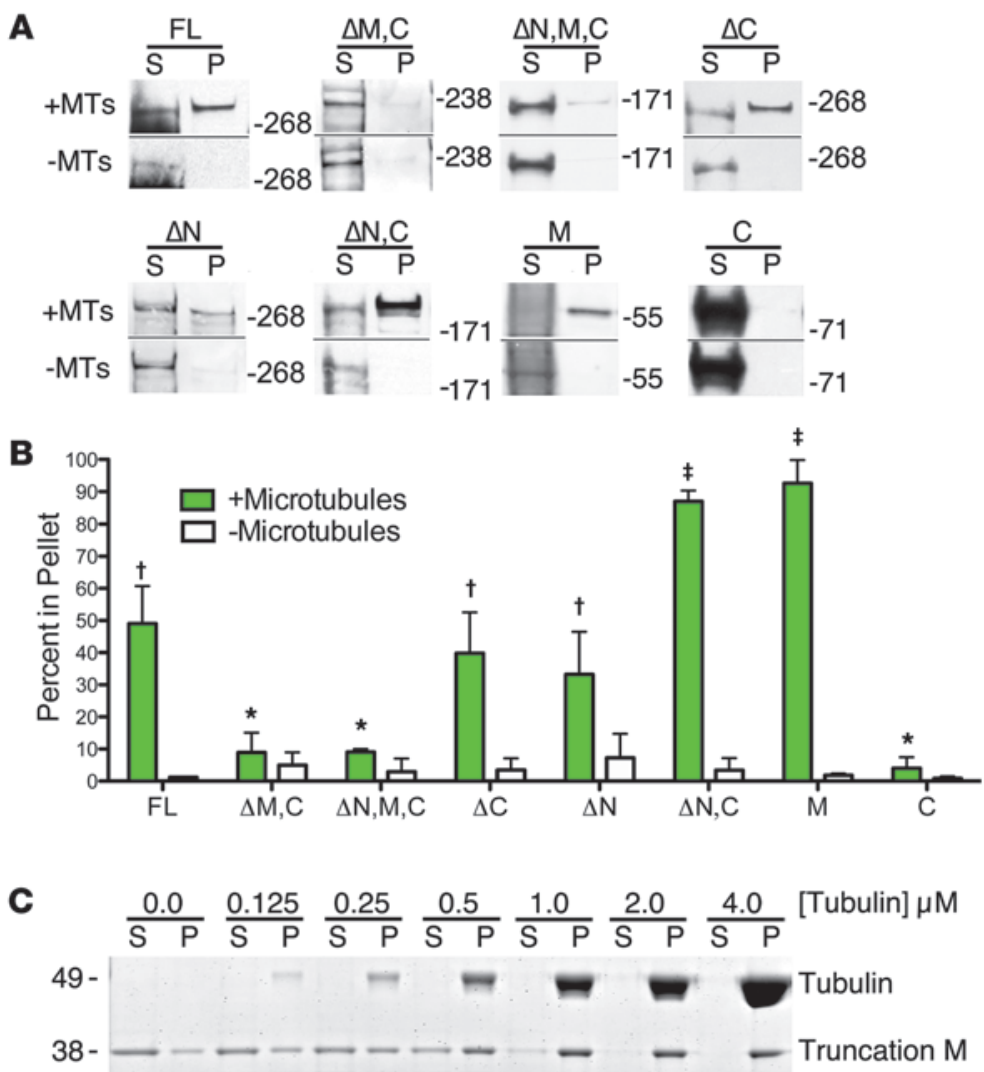

D

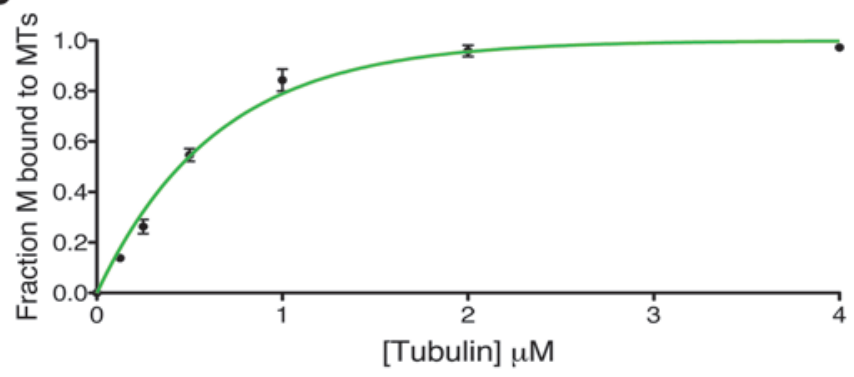

Figure 7

CEP290 region M mediates direct microtubule binding. (A) Microtubule cosedimentation assays for in vitro transcribed and translated CEP290 truncations. The supernatants (S) and microtubule pellets $(P)$ are shown for assays performed both with $(+\mathrm{MT})$ and without $(-\mathrm{MT})$ microtubules. (B) Percentage of each truncation cosedimenting with microtubules. Data are presented as mean $\pm \mathrm{SD}, n=3$. Symbols indicate significance as follows: ${ }^{*} P<0.05$; ${ }^{\dagger} P<0.05$; $\ddagger P<0.05$. (C) Coomassie-stained gel of microtubule cosedimentation assays performed with purified CEP290 truncation $\mathrm{M}$ and increasing concentrations of microtubules. The supernatants and microtubule pellets are shown. (D) Binding curve of microtubules cosedimentation assays. The fraction of truncation $M$ present in the pellet in the absence of microtubules was subtracted from all data points. Data are presented as means $\pm \mathrm{SD}, n=3$.

The rd16 mouse Cep290 gene encodes a version of the protein deficient in microtubule binding. The rd16 mouse is a retinal disease model of CEP290 deficiency characterized by a rapid and near-complete degeneration of photoreceptors. The rd16 Cep290 gene encodes a protein containing an in-frame deletion of 298 amino acids (14) that significantly overlaps the region of human CEP290 we identified as being critical for microtubule binding (Figure 10A).

We generated 2 truncation mutants of human CEP290 containing either the region of the microtubule-binding domain deleted in the $r d 16$ mouse or the region of the microtubule binding domain spared by the mouse deletion (Figure 10A). To our surprise, when overexpressed in hTERT-RPE1 cells, both truncations displayed a diffuse localization pattern indicative of a primarily cytosolic localization (Figure 10B). In neither case was any significant colocalization with the microtubule network observed. We confirmed that neither of these constructs was capable of associating with microtubules by subjecting them to microtubule cosedimentation assays (Figure 10C). Neither construct was found to significantly cosediment with microtubules (Figure 10D), indicating both that the rd16 deletion perturbs microtubule bind-

cantly increased, by more than $25 \%$, compared with cells treated with the control vector (Figure 8, C and D). These increases were only observed in cells maintained in medium supplemented with serum, a condition in which CEP290 is normally inhibited (21), implying that these regulatory domains act through the same pathway that mediates normal CEP290 inhibition. It is also interesting to note that dysregulation of CEP290 by overexpression of either regulatory region was sufficient to initiate aberrant primary cilium formation, suggesting that there is no further downstream regulation of ciliogenesis beyond CEP290.

Interestingly, in both the serum-starved and serum-fed state, it was noted that occasional cells transduced with the $\mathrm{N}$ terminus of CEP290 appeared to produce multiple ciliary axonemes at the same centrosome (Figure 8A and 9, A-C). These axonemes always emanated from a single focus of pericentrin (Figure $8 \mathrm{~B}$ ) and were often found at $90^{\circ}$ to each other. In some, but not all cases, one or more of these axonemes costained with ARL13B (Figure 9A), a protein associated with the ciliary membrane (30), indicating that at least some of these multi-axoneme structures were fully formed cilia. ing and that microtubule binding is conferred by a larger portion of the CEP290 gene than was included in either of our truncations.

To confirm that $r d 16$ Cep290 was in fact deficient in microtubule binding, we subjected brain lysates from WT and $r d 16$ mice to microtubule cosedimentation assays (Figure 10E). WT Cep290 showed very significant microtubule association, with roughly $60 \%$ of the protein associating with microtubules (comparable to fulllength human CEP290; Figure 7B), while rd16 Cep290 was found to be completely deficient in microtubule binding (Figure 10F).

The rd16 mouse is deficient in cilium formation and structure. While the retinal phenotype of the $r d 16$ mouse has been well documented (38), no cellular phenotype regarding primary cilium formation or structure has yet been reported. To investigate the effect that ablation of Cep290 microtubule binding might have on primary cilium formation, we assayed primary dermal fibroblasts from $r d 16$ and WT mice for deficiencies in cilium formation and structure. In both serum-starved and serum-fed conditions, $r d 16$ fibroblasts were found to be significantly deficient in primary cilium formation, with roughly $50 \%$ fewer cells forming cilia than WT controls 
A
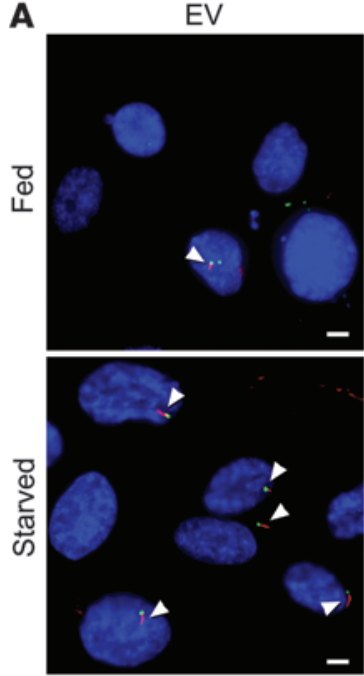

C
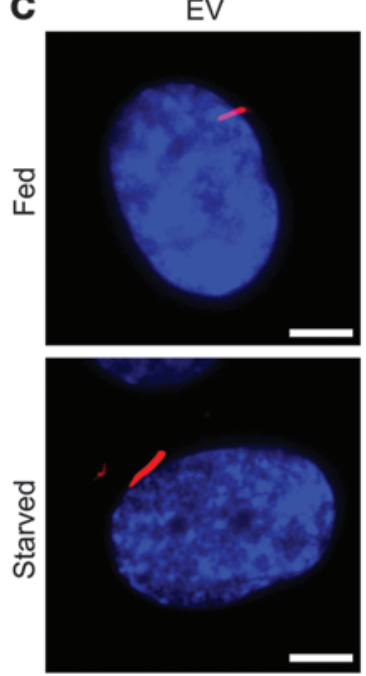

$\mathrm{N}$
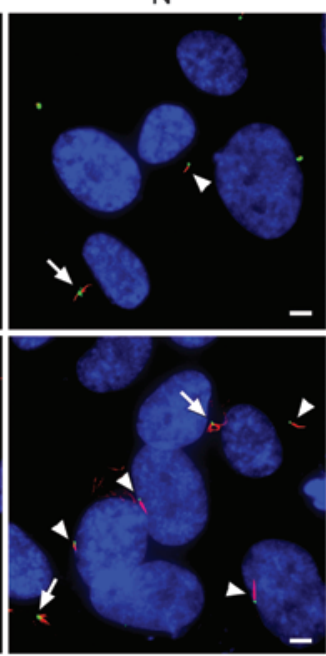

$\mathrm{N}$
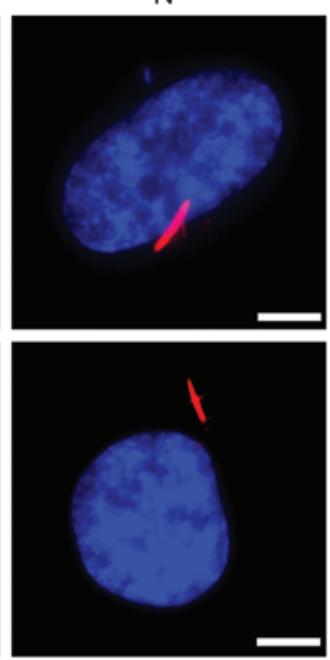
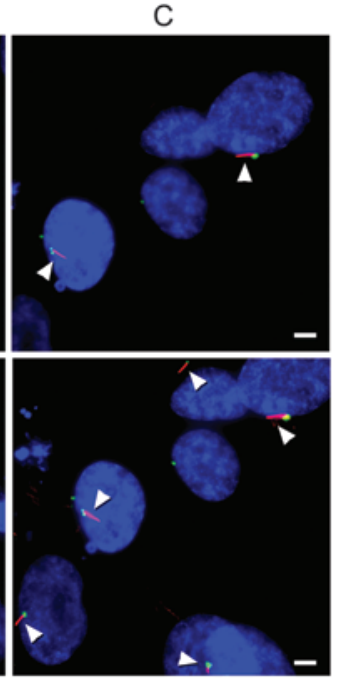

C
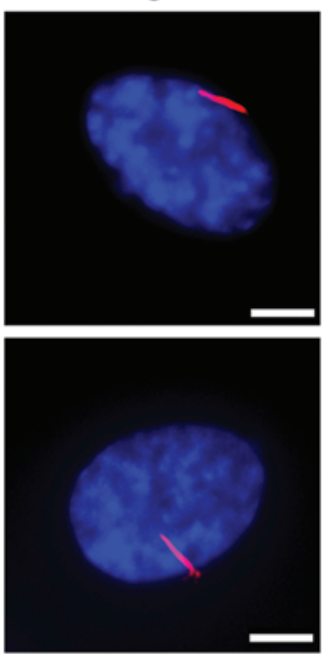

B
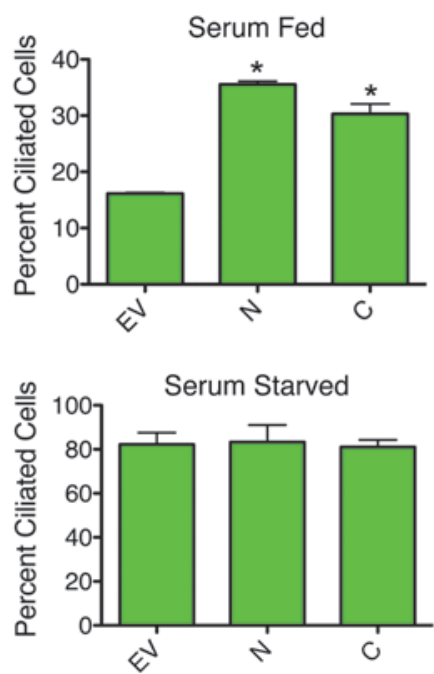

D
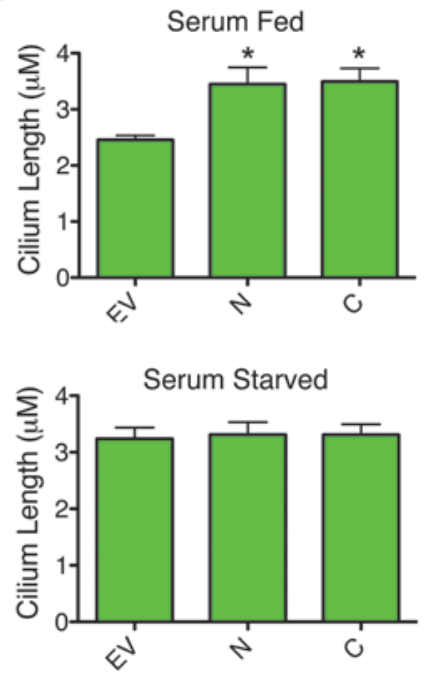

Figure 8

Overexpression of either $\mathrm{N}$ - or C-terminal regulatory region of CEP290 ablates normal CEP290 inhibition. (A) Fluorescence microscopy fields of hTERT-RPE1 cells transduced with lentiviral empty vector (EV), or vectors encoding either the N (aa 1-580) or C terminus (aa 1966-2479) of CEP290. Cells were stained for acetylated $\alpha$-tubulin (red) and pericentrin (green) to detect primary cilia and with DAPI. Arrowheads indicate primary cilia. Arrows indicate cells with multiple axonemes originating from the same focus of pericentrin. Scale bars: $10 \mu \mathrm{m}$. (B) Percentage of lentivirus-treated cells forming primary cilia. Data are presented as mean $\pm \mathrm{SD}, n=3.100$ cells were counted per experiment. (C) Fluorescence microscopy images of hTERT-RPE1 cells transduced with lentiviral vectors as in A. Cells were stained for acetylated $\alpha$-tubulin to detect primary cilia and with DAPI. Scale bars: $5 \mu \mathrm{m}$. (D) Average primary cilium length for hTERT-RPE1 cells as in C. Data are presented as mean \pm SD, $n=3$. A total of at least 150 cilia were measured per condition. ${ }^{*} P<0.05$.

(Figure 11, C and D). The cilia produced by $r d 16$ fibroblasts were also found to be more than $25 \%$ shorter than those produced by WT fibroblasts (Figure 11, A and B), further suggesting that the microtubule binding functionality of CEP290 is critically important in the maintenance and formation of the primary cilium and, when disrupted, capable of causing severe retinal disease.

\section{Discussion}

CEP290 plays a critical role in the formation and function of the primary cilium and, when mutated, is responsible for a variety of devastating human diseases (16). Our understanding of the protein's function has been, until now, inadequate to explain how disruption of CEP290 might lead to human pathology (27). The findings presented here begin to implicate CEP290 as a vital structural and regulatory element of the ciliary transition zone, elucidate some of its molecular functionality, and place it at the center of the critically important and disease-relevant pathways of ciliogenesis and IFT.

CEP290 as a bridge between the ciliary membrane and the microtubule axoneme. To date, how CEP290 functions as a component of the ciliary Y-links has been unclear. Here we have shown that CEP290 is capable of directly binding to both cellular membranes and microtubules, possibly anchoring the two to each other and likely playing a key structural role in the maintenance of the cilium. Fur- 

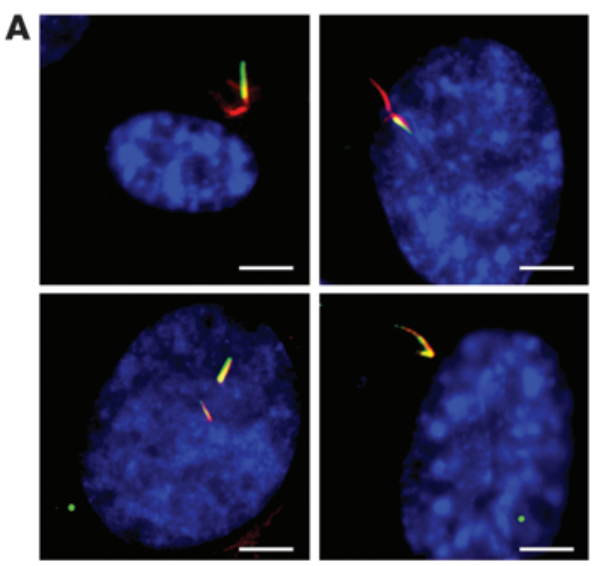

Ac. Tubulin

ARL13B
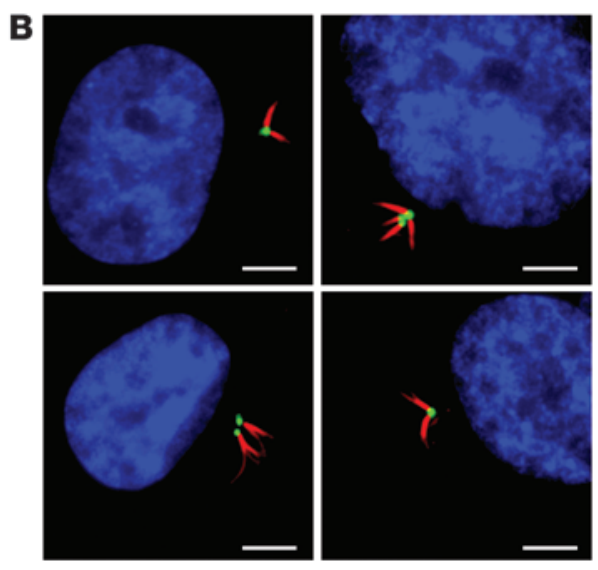

Ac. Tubulin

Pericentrin

\section{C}

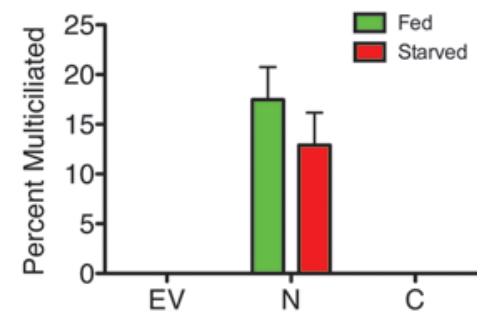

\section{Figure 9}

Overexpression of the $\mathrm{N}$-terminal regulatory region of CEP290 results in multiple ciliary axonemes. (A and B) Fluorescence microscopy images of hTERT-RPE1 cells that formed multiple cilia after transduction with lentiviral vector encoding the N terminus of CEP290. Cells were stained for either ARL13B or pericentrin and for acetylated $\alpha$-tubulin and with DAPI (blue). Scale bars: $5 \mu \mathrm{m}$. (C) Percentage of lentivirus-transduced hTERTRPE1 cells forming multiple cilia. At least 100 cells were counted per experiment. Data are presented as mean $\pm \mathrm{SD}, n=3$.

thermore, we have described the particular domains of CEP290 responsible for mediating these specific functions. The $\mathrm{N}$ terminus of the protein, containing a highly conserved amphipathic helix, mediates CEP290's membrane-binding activity. Such helices have previously been shown to be critical in mediating robust, reversible interactions between various membrane-binding proteins and cellular membranes (35). The role of this domain in membrane association is corroborated by recent evidence that this same region of the protein interacts with multiple components of the BBSome, a complex of proteins that functions to sort membrane proteins to the primary cilium (39). Multiple experiments determined that a region near the $\mathrm{C}$ terminus of CEP290, encompassing much of the protein's myosin-tail homology domain, was necessary and sufficient to mediate microtubule binding. Myosin-tail homology domains have been reported to be critical in mediating the microtubule-binding activity of several other cytoskeleton-associated proteins $(40,41)$.

The location of these 2 functional domains at opposite ends of CEP290 immediately suggests a potential structural role for the protein, anchoring the ciliary membrane to the axoneme at a fixed distance. Our data also indicate that, in addition to binding microtubules, CEP290 is capable of mediating their acetylation and bundling, 2 hallmarks of the microtubules that make up the ciliary axoneme (42), suggesting that CEP290 might play an important role in the stabilization, bundling, and organization of microtubules during ciliogenesis.

A mechanism for the inhibition of CEP290. An overarching theme that emerged from our analysis of CEP290's membrane- and microtubule-binding activities was that the full-length protein exhibited attenuated activity when compared with truncation mutants lacking the $\mathrm{N}$ or $\mathrm{C}$ terminus. Confirming a role for these domains in the regulation of the protein's function and ciliogenesis, we found that overexpression of either domain interfered with the normal regulation of CEP290 and was sufficient to initiate aberrant primary cilium formation, suggesting that there is no further downstream regulation of ciliogenesis beyond
CEP290. Overexpression of the regulatory regions in serumstarved cells, where CEP290 is known to be relieved of inhibition, did not result in any increase in CEP290 activity, implying that these regulatory domains act through the same pathway that mediates normal CEP290 inhibition.

These data present at least 2 possibilities for a mechanism of CEP290 regulation. Both regulatory loci might be acted upon by extraneous inhibitory factors to mediate CEP290 inhibition. In fact, it has been shown that the protein CP110 acts as just such an inhibitory factor, binding to the N terminus of CEP290 and inhibiting protein activity $(15,16)$. Accordingly, we found that, in some cells, overexpression of CEP290's N terminus led to the growth of multiple ciliary axonemes. This is consistent with what would be expected upon competition between endogenous CEP290 and the overexpressed N-terminal fragment for CP110, normally removed from only one end of the mother centriole to initiate ciliogenesis (43). Nonspecific depletion of CP110 from both ends of both centrioles by the overexpressed $\mathrm{N}$ terminus could have resulted in the growth of multiple ciliary axonemes in the cells we observed.

Our data cannot, however, be fully explained by this mechanism alone. If inhibition were solely dependent on the binding of a finite pool of endogenous inhibitory factor, then overexpression of the full-length protein should result in competition for the inhibitory factor and only minimal, if any, observed inhibition of the overexpressed protein. Additionally, we would not expect to see inhibition of the full-length construct in in vitro assays where inhibitory factors should not be present at meaningful concentrations. In all our experiments assessing protein activity, we found that full-length CEP290 was substantially inhibited compared with truncation mutants lacking the novel inhibitory regions, arguing against extraneous factors such as CP110 being solely responsible for CEP290 regulation.

We propose an alternative model for CEP290 regulation that is more consistent with our findings and with previous data showing that both the $\mathrm{N}$ and $\mathrm{C}$ termini of CEP290 are capable of binding both themselves and each other (26). Perhaps the 2 novel regula- 
A

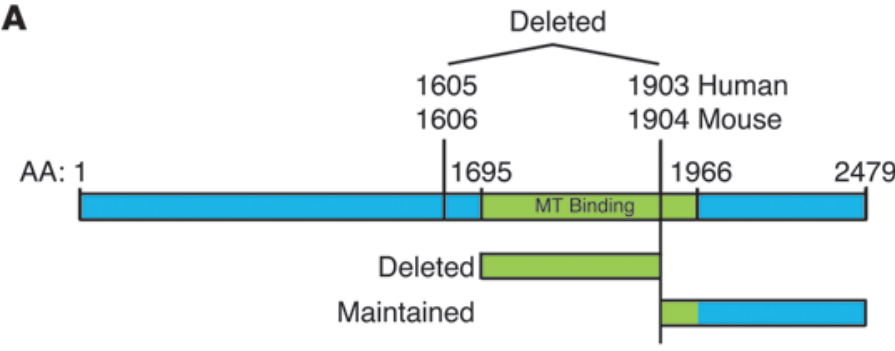

B
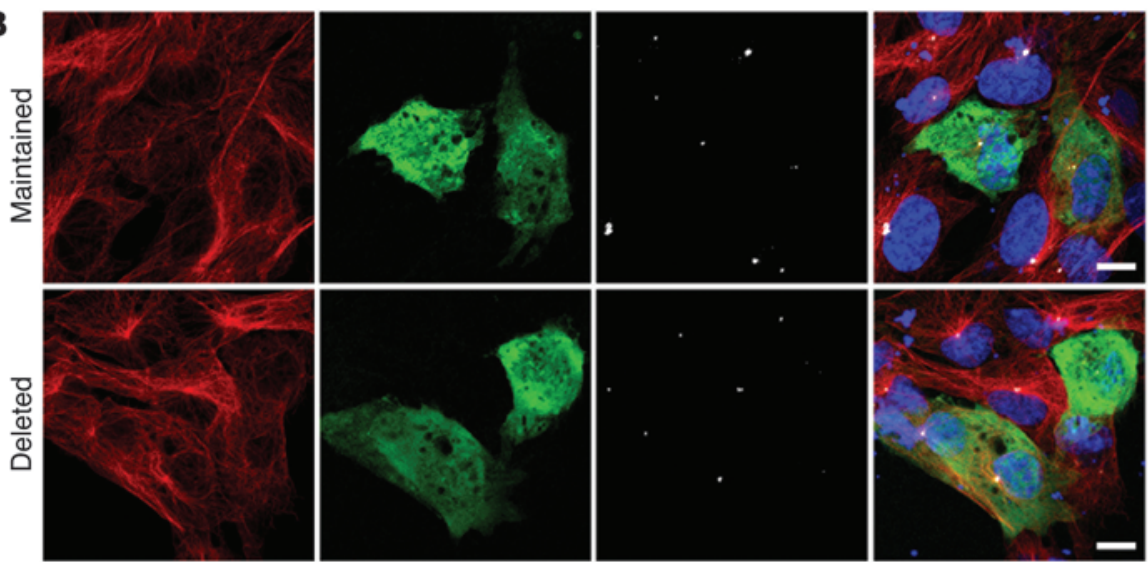

C

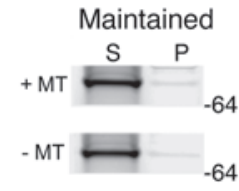

Deleted

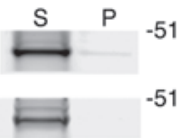

D

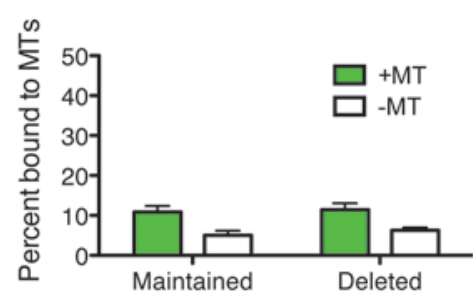

E

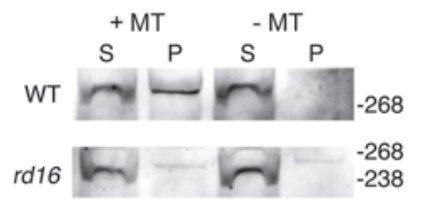

$\mathbf{F}$

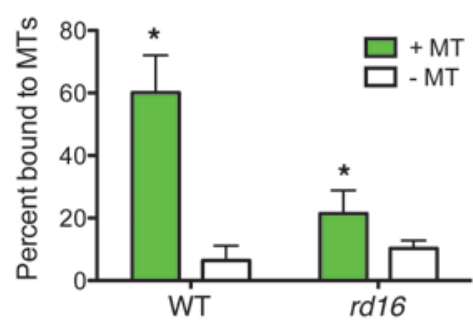

\section{Figure 10}

An in-frame deletion in Cep290 in the rd16 mouse ablates Cep290's microtubule binding activity. (A) Schematic representation of the microtubule-binding region of human CEP290 in relation to the rd16 mouse deletion (14). Truncations of CEP290 representing the part of the microtubule-binding region deleted in the $r d 16$ mouse and the part of the microtubule-binding region maintained in the rd16 mouse were created as shown. (B) Confocal fluorescence microscopy images showing the localization pattern of GFP-tagged maintained and deleted CEP290 truncations. Cells were stained for $\alpha$-tubulin (red) and pericentrin (white) and with DAPI (blue). Scale bars: $10 \mu \mathrm{m}$. (C) Microtubule cosedimentation assays for in vitro transcribed and translated maintained and deleted CEP290 truncations. The supernatant and microtubule pellet fractions are shown in assays performed both with and without microtubules. (D) Percentage of each truncation cosedimenting with microtubules. Data are presented as mean $\pm \mathrm{SD}, n=2$. (E) Microtubule cosedimentation assays performed on full-length WT and rd16 Cep290 from mouse brain homogenate. The supernatant and microtubule pellet fractions are shown in assays performed on samples induced to polymerize microtubules and samples treated to prevent microtubule polymerization. (F) Percentage of WT and rd16 Cep290 cosedimenting with microtubules. Data are presented as mean $\pm \mathrm{SD}, n=3$. Asterisks indicate statistical significance over $-\mathrm{MT}$ samples. ${ }^{\star} P<0.05$.

tory regions of the protein cooperate to inhibit CEP290 function, binding to each other and causing a conformational change in the protein, stabilized by the binding of CP110, that obscures important functional domains and decreases protein function (Figure 12). Thus, the overexpression of either regulatory domain would saturate endogenous CEP290 regulatory domains, resulting in a paradoxical increase in protein function, and the full-length protein, of its own accord, would be expected to display innate inhibition regardless of experimental conditions. These are exactly the results we observed in all of our experiments.

Interestingly, this mechanism of inhibition has been described for a variety of other proteins, such as Vinculin, Ezrin, and the ERM domain proteins, all of which act to link the cell membrane to the actin cytoskeleton $(44,45)$. These proteins depend on amphipathic helices for their membrane-binding activity, and these helices have also been shown to act in the inhibition of these proteins, binding to and obscuring their actin-binding domains (35). It is plausible that this mechanism of autoinhibition, conserved among membrane-to-actin cytoskeleton- bridging proteins, is operative and conserved for membrane to microtubule-bridging proteins as well. CEP290 would be the first protein in this class shown to rely upon this mechanism of inhibition. This model is also supported by observations made in the $r d 16$ mouse, such as the apparent increased affinity of $r d 16$ Cep290, compared with WT, for RPGR (14). The $r d 16$ deletion of the microtubule binding/regulatory region may thus result in decreased autoinhibition and a higher affinity of Cep290 for its interacting partners, as was observed.

Implications for buman disease. As we continue to learn more about the key functional domains of CEP290, we will begin to better understand how mutations in particular protein domains contribute to pathology. The microtubule-binding function of CEP290 is clearly of critical importance to the function of the protein on a cellular level and, as we have shown, critical in disease. The rd16 mouse Cep290 gene was found to encode a version of the protein completely deficient in microtubule binding, resulting in deficiencies in primary cilium formation and structure in cultured fibroblasts. While $r d 16$ photorecep- 
A
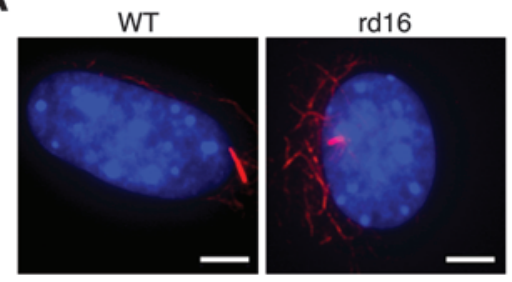

C
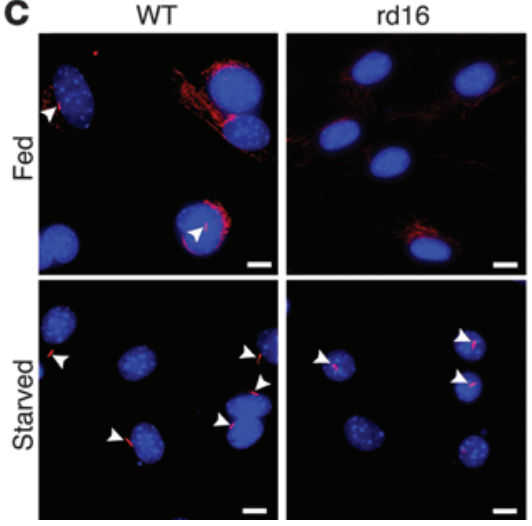

B

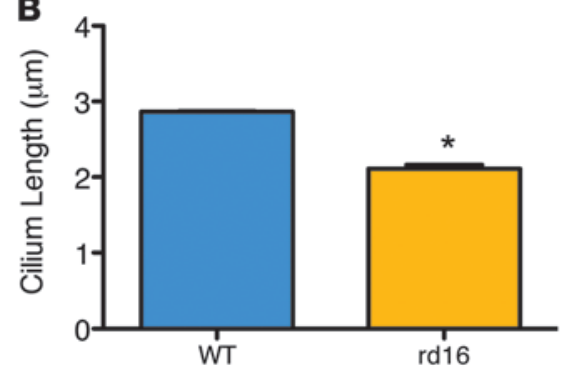

D

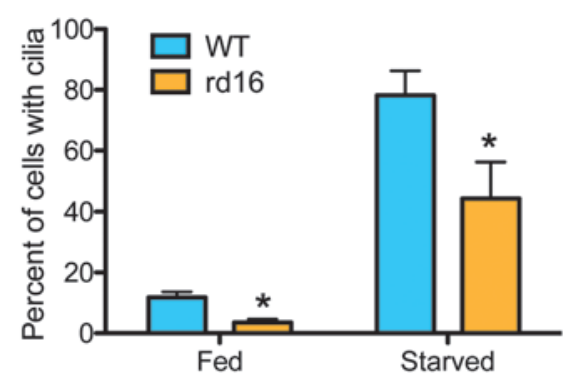

\section{Figure 11}

rd16 mouse fibroblasts are deficient in primary cilium formation. (A) Fluorescent microscopy images of serum-starved WT and rd16 primary dermal fibroblasts stained for acetylated $\alpha$-tubulin (red) to identify primary cilia and stained with DAPI (blue). Scale bars: $5 \mu \mathrm{m}$. (B) Average cilium length of serum-starved WT and $r d 16$ primary dermal fibroblasts. Quantification was based on separate experiments on fibroblasts coming from 3 different animals per genotype. At least 50 cilia were measured per experiment, and a total of 400 cilia were measured for both the WT and $r d 16$ fibroblasts. Data are presented as mean $\pm \mathrm{SD}, n=5$. (C) Fluorescence microscopy fields of WT and $r d 16$ primary dermal fibroblasts stained for acetylated $\alpha$-tubulin (red) to identify primary cilia and stained with DAPI (blue). Fibroblasts were grown in medium with (fed) or without (starved) serum. Arrowheads indicate primary cilia. Scale bars: $10 \mu \mathrm{m}$. (D) Percentage of WT and rd16 primary dermal fibroblasts that form primary cilia in serum-fed and serum-starved conditions. Quantification was based on separate experiments on fibroblasts coming from 3 different animals per genotype. At least 100 cells were counted per experiment. Data are presented as mean $\pm \mathrm{SD}, n=5$. ${ }^{*} P<0.05$.

tors do seem capable of forming connecting cilia, the function and maintenance of the connecting cilium and photoreceptor outer segment is perturbed in these animals, resulting in dramatic and rapid retinal degeneration, implying that deficiencies in CEP290 microtubule binding can lead to marked pathology. Over 24 unique mutations identified in human CEP290 patients map to the microtubule binding domain we report here (Figure 1A). Almost all of these mutations are expected to have truncating effects on the protein (28), which, as in the $r d 16$ mouse, would result in significant deficiencies in microtubule binding, potentially explaining the mechanism underlying the disease phenotype seen in these individuals.

Perhaps most interesting, however, are the implications that the discovery of novel regulatory domains of CEP290 has for the development of therapeutic interventions. The extension, to CEP290 patients, of recombinant adeno-associated virus-based (AAV-based) therapeutics, which have proven safe and effective in the treatment of another genetic cause of LCA (43), has been hindered by CEP290's large size, precluding it from packaging in
AAV (44). Truncation mutants of CEP290 lacking the inhibitory domains we have described but maintaining the other functional regions of the protein may exhibit normal, or even enhanced, CEP290 function, while at the same time being small enough to fit in AAV. The delivery of such a therapeutic to terminally differentiated tissues, such as the retina, where permanent activation of CEP290 would not be expected to be problematic, might prove effective in the treatment of CEP290-related diseases such as LCA.

\section{Methods}

Plasmid construction. CEP290 truncations were generated by PCR amplification using Gateway (Invitrogen) cloning-compatible primers (Supplemental Table 1) from a human codonoptimized CEP290 plasmid synthesized by DNA 2.0. Amplified products were cloned into pDONR221 (Invitrogen) by Gateway cloning to generate entry clones. For cell transfection and in vitro transcription and translation assays, entry clones were shuttled into the plasmid pcDNA-DEST53 (Invitrogen) by Gateway LR clonase reactions to create $\mathrm{N}$-terminally tagged GFP fusions. For bacterial expression, entry clones were shuttled into pDest-527 (a gift of Dominic Esposito, Frederick National Laboratory for Cancer Research, Frederick, Maryland, USA; Addgene plasmid \#11518) to create $\mathrm{N}$-terminally tagged $6 \mathrm{xHis}$ fusions. For lentivirus production, entry clones were shuttled into pLXnGFP, a modified version of pLX302 (a gift of David Root, The Broad Institute, Cambridge, Massachusetts, USA; Addgene plasmid \#25896), to create $\mathrm{N}$-terminally tagged GFP fusion lentivirus production plasmids. Restriction digest and DNA sequencing were used to confirm the integrity of each expression construct.

Cell culture and treatments and lentivirus production. WT and $r d 16$ mouse primary dermal fibroblasts and 293T cells were grown in DMEM supplemented with $10 \%$ FBS. hTERT RPE-1 cells were grown in DMEM:F12 supplemented with $10 \%$ FBS and $0.075 \%$ sodium bicarbonate. All cells were grown at $37^{\circ} \mathrm{C}$ in a humidified $5 \% \mathrm{CO}_{2}$ atmosphere. Transfections were performed with FuGENE 6 reagent (Promega) according to the manufacturer's protocol. Cells were induced to form primary cilia by serum starvation with Opti-MEM I (Invitrogen) for 48-72 hours.

Lentiviral vectors were produced by transfection of $80 \%$ confluent $293 \mathrm{~T}$ cells in T25 culture flasks with $1 \mu \mathrm{g}$ of lentivirus construct, $750 \mathrm{ng}$ of PsPAX2 packaging plasmid, and 250 ng of PMD2.G envelope plasmid. Medium was replaced after 24 hours, and lentiviral supernatants were harvested at 48 and 72 hours, combined, filtered through a $0.45-\mu \mathrm{m}$ filter, and snap-frozen at $-80^{\circ} \mathrm{C}$. For lentivirus transduction, hTERT RPE- 1 cells were plated in medium containing $8 \mu \mathrm{g} / \mathrm{ml}$ polybrene. Filtered medium containing the appropriate lentivirus particles was added, and 24 hours after transduction, cells were switched to selective medium containing $10 \mu \mathrm{g} / \mathrm{ml}$ puromycin.

Primary dermal fibroblast isolation. Primary dermal fibroblasts were isolated by washing neonatal mouse skin in $70 \%$ ethanol followed by 5 washes in 


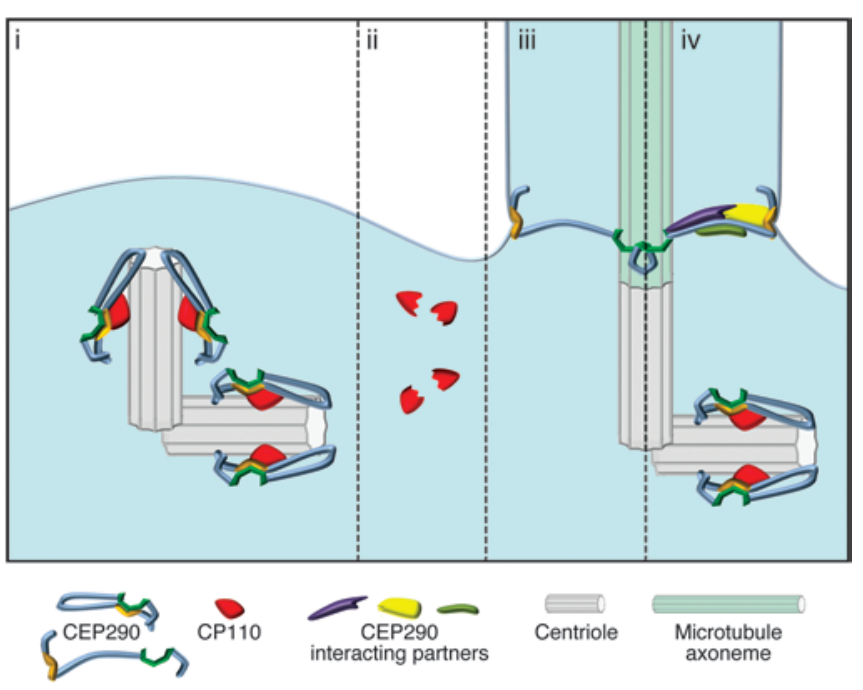

PBS. The skin was minced in a culture vessel, covered with DMEM supplemented with $10 \% \mathrm{FBS}$, penicillin, and streptomycin, and incubated at $37^{\circ} \mathrm{C}$ in a humidified $5 \% \mathrm{CO}_{2}$ atmosphere. One week after harvesting, the skin was removed and discarded and fibroblasts were passaged into a larger vessel.

Antibodies, immunofluorescence, and immunoblotting. Antibodies used in this study were rabbit anti-human CEP290 (Abcam, ab105383), rabbit anti-mouse Cep290 (Abcam, ab128231), mouse anti- $\alpha$ tubulin (Abcam, AB7291), rabbit anti-pericentrin (Abcam, AB4448), mouse anti-GFP (Roche 11814460 001), mouse anti-bovine serum albumin (Thermo, MA5-15238), rabbit anti-6xHis (Abcam, AB1187), rabbit anti-GAPDH (Sigma-Aldrich, SAB2103104), mouse anti-Na/K ATPase $\alpha-1$ (Novus, NB300-146), mouse anti-Lamin A/C (Sigma-Aldrich, SAB4200236), mouse anti-acetylated $\alpha$-tubulin (Sigma-Aldrich, T7451), rabbit antiLAMP2 (Novus, NBP1-71692), rabbit anti-annexin A2 (Cell Signaling, 8235), rabbit anti-ARL13B (Proteintech, 17711-1-AP), HRP-conjugated goat anti-mouse (GE, NA931V), HRP-conjugated goat anti-rabbit (GE, NA934V), Cy5 conjugated goat anti-rabbit (KPL, 072-02-15-06), and Alexa Fluor 594-conjugated goat anti-mouse (Invitrogen, A1100S).

For immunofluorescence, cells were grown in chamber slides and fixed with $3 \% \mathrm{PFA}$ in PBS for 20 minutes at $37^{\circ} \mathrm{C}$. Cells were permeabilized with $1 \%$ Triton X-100 in PBS for 5 minutes and blocked in 2\% BSA in PBS for 30 minutes prior to incubation with primary antibody. Secondary antibodies used were donkey anti-mouse or anti-rabbit, conjugated to Cy5 or Alexa Fluor 594. Slides were mounted in mounting medium containing DAPI. Confocal imaging was performed with an LSM510 META NLO laser scanning confocal on a Zeiss Axiovert 200M inverted microscope using a Plan-Apo $\times 63 / 1.4$ oil objective and the LSM510 4.2 software. Laser lines used were $488 \mathrm{~nm}$ (for green labeling, from argon laser), $543 \mathrm{~nm}$ (for red labeling, HeNe laser), $633 \mathrm{~nm}$ (for cy5 channel, HeNe laser), and $740 \mathrm{~nm}$ (for DAPI channel, from a Coherent Chameleon Tunable 2 Photon Laser). For normal fluorescence microscopy, slides were imaged using an Axio Imager.M2 microscope using either an EC Plan-Neofluar ×40/0.75 M27 or an EC Plan-Neofluar $\times 63 / 1.25$ Oil M27 objective and captured using an AxioCamMR3 camera and the AxioVs40 software, version 4.8.2.0. Primary cilium length was measured using the same AxioVs40 software.

For immunoblotting, samples were subjected to SDS-PAGE and transferred to nitrocellulose membrane using standard techniques. Membranes were blocked in $5 \%$ nonfat milk for 1 hour at room temperature and subsequently incubated in primary antibody overnight at $4^{\circ} \mathrm{C}$. Membranes were washed 3 times with PBST (0.1\% Tween-20 in PBS), incubated with HRP-conjugated

\section{Figure 12}

A speculative model for CEP290 activity at the primary cilium. CEP290 is maintained in a closed, inhibited state by its $\mathrm{N}$ and $\mathrm{C}$ termini and CP110 during the cell cycle (i). Upon degradation of CP110 at the mother centriole (ii), a conformational change in the protein frees and activates CEP290's membrane-binding (orange) and microtubule-binding (green) domains (iii), and CEP290 is able to recruit additional interacting partners to initiate IFT and ciliogenesis (iv).

secondary antibody for 1 hour at room temperature, washed, and developed using ECL2 reagent (Pierce), and scanned on a Typhoon 9400 instrument (GE). Immunoblots were densitometrically quantified using ImageJ $1.44 \mathrm{p}$.

Membrane flotation, membrane protein fractionation, and vesicle immunoprecipitation. Cultured cells were washed 3 times with ice-cold PBS. Cells were resuspended in a $250 \mathrm{mM}$ sucrose solution containing $4 \mathrm{mM} \mathrm{Immid-}$ azole and a protease inhibitor cocktail and passaged through a 25 -gauge needle 20 times. The resulting lysate was centrifuged at $1,000 \mathrm{~g}$ for $10 \mathrm{~min}$ utes at $4^{\circ} \mathrm{C}$ to pellet nuclei and unlysed cells, and the resulting postnuclear supernatant was centrifuged at $100,000 \mathrm{~g}$ for 60 minutes at $4^{\circ} \mathrm{C}$ to pellet the membrane-enriched fraction.

Membrane flotation was performed by resuspending the membraneenriched fraction in $250 \mu \mathrm{l}$ of $80 \%$ sucrose in PBS. This solution was added to the bottom of a $2-\mathrm{ml}$ centrifuge tube and overlaid with $1.5 \mathrm{ml}$ of $50 \%$ sucrose in PBS and with $250 \mu \mathrm{l}$ of $5 \%$ sucrose in PBS. Sucrose gradients were then centrifuged at $100,000 \mathrm{~g}$ for 16 hours at $4^{\circ} \mathrm{C}$. Equal fractions were subsequently taken from the top to the bottom of the gradient and analyzed by Western blotting as indicated.

Peripheral membrane proteins were extracted from membrane preparations by resuspending and incubating the membrane-enriched fraction of cultured cells in a high $\mathrm{pH}$ buffer $\left(100 \mathrm{nM} \mathrm{Na}_{2} \mathrm{CO}_{3}\right.$, $\left.\mathrm{pH} 11.3\right)$ for $30 \mathrm{~min}$ utes at $4^{\circ} \mathrm{C}$. The membranes were pelleted at $100,000 \mathrm{~g}$ for 60 minutes at $4^{\circ} \mathrm{C}$, and the resulting supernatant was saved as the peripheral membrane fraction. Integral membrane proteins were subsequently extracted by resuspending and incubating the resulting membrane pellet in $4 \%$ Triton-X 100 in PBS for 30 minutes at $4^{\circ} \mathrm{C}$.

Vesicle immunoprecipitation was performed on postnuclear supernatants of hTERT-RPE1 cells expressing various CEP290 truncations. A sample of the total postnuclear extract was saved as the input fraction. Protein G Dynabeads (Invitrogen) were washed and incubated with $2 \mu \mathrm{g}$ of anti-ARL13B antibody for 20 minutes at room temperature, magnetically collected, and washed in PBS. Then $350 \mu$ l of postnuclear supernatant was added to the antibody-Dynabead complex and incubated with gentle agitation for 20 minutes at room temperature. The beads and immunoprecipitated complexes were magnetically collected and washed 3 times with PBS. A sample of the unbound fraction was saved, and the immunoprecipitated material was eluted by resuspension in $4 \times$ SDS PAGE sample buffer.

Subcellular fractionation. Subcellular fractionation was performed using the QProteome Cell Compartment Kit (QIAGEN) according to the supplied protocol. 
Recombinant protein expression and purification. 6xHis-tagged CEP290 truncation $\mathrm{M}$ and $\mathrm{N}$ were expressed from pDest-527 in E. coli BL21(DE3) pLysS (Invitrogen) and purified using the Ni-NTA Fast Start Kit (QIAGEN) according to the manufacturer's protocol. Purified protein products were subjected to SDS-PAGE and stained with Coomassie Brilliant Blue to assess purity and determine protein concentration.

Liposome flotation assay. $100 \mathrm{~nm}$ liposomes (total lipid concentration of 5 $\mathrm{mg} / \mathrm{ml}$ in PBS) with a lipid composition of a 60:40 molar ratio of phosphatidylserine to cholesterol were purchased from Encapsula NanoSciences and used within 2 weeks of formulation. $20 \mu \mathrm{l}(1.5 \mu \mathrm{g})$ of recombinant CEP290 truncation N, or an equivalent amount of BSA, was incubated with $230 \mu \mathrm{l}$ of liposomes or an equal volume of PBS alone at $37^{\circ} \mathrm{C}$ for 30 minutes. Each reaction was then mixed 1:1 with a solution of $80 \%$ sucrose in PBS and added to the bottom of a 2-ml ultracentrifuge tube. Reactions were overlaid with $1.3 \mathrm{ml}$ of $30 \%$ sucrose in PBS and with $200 \mu \mathrm{l}$ of PBS and centrifuged at $100,000 \mathrm{~g}$ for 90 minutes at $30^{\circ} \mathrm{C}$. Five $400-\mu \mathrm{l}$ fractions were taken, starting at the top of sucrose gradient, and equal amounts of each were analyzed by SDS-PAGE and Western blotting.

In vitro transcription and translation reactions. Plasmid DNA was transcribed and translated using the TNT T7 reticulocyte lysate system (Promega) according to the manufacturer's protocol.

Microtubule polymerization. Pure bovine tubulin (Cytoskeleton) was diluted 1:1 in BRB80 buffer ( $80 \mathrm{mM}$ Pipes, $1 \mathrm{mM} \mathrm{MgCl} 2,1 \mathrm{mM}$ EGTA, $\mathrm{pH}$ 6.8) and cleared of insoluble material by centrifugation at $20,000 \mathrm{~g}$ for 10 minutes. The soluble fraction was supplemented with $1 \mathrm{mM} \mathrm{GTP}$ and incubated at $37^{\circ} \mathrm{C}$ for 15 minutes to polymerize microtubules. The polymerized microtubules were treated with $10 \mu \mathrm{M}$ taxol and incubated at room temperature for an additional 15 minutes to stabilize the microtubules. Microtubules were pelleted at $48,000 \mathrm{~g}$ for 30 minutes at $30^{\circ} \mathrm{C}$ and resuspended in BRB80 supplemented with $1 \mathrm{mM}$ GTP and $10 \mu \mathrm{M}$ taxol. Microtubules were used within 1 week of preparation.

Microtubule-binding assays. Crude TNT T7 reaction products were diluted 1:1 in BRB80 supplemented with $1 \mathrm{mM} \mathrm{GTP}$ and $10 \mu \mathrm{M}$ taxol. The diluted products were then incubated at $30^{\circ} \mathrm{C}$ for 30 minutes in either the presence or absence of pure, prepolymerized microtubules. Reactions were centrifuged through a $40 \%$ sucrose cushion at $48,000 \mathrm{~g}$ for 30 minutes, the supernatant was collected, and the pellet washed once with warm BRB80 and resuspended in $1 \times$ SDS PAGE sample buffer. Both fractions were subjected to SDS-PAGE and transferred to nitrocellulose. The presence of tubulin in the pellets was confirmed by Ponceau staining.

For microtubule-binding assays performed on mouse brain homogenate, $0.5 \mathrm{~g}$ of mouse brain was mechanically homogenized in $0.5 \mathrm{ml}$ of $1 \% \mathrm{NP} 40$ in BRB80 buffer containing a protease inhibitor cocktail. The homogenate was cleared of insoluble material by centrifugation at 48,000 $\mathrm{g}$ for $30 \mathrm{~min}$ utes at $4^{\circ} \mathrm{C}$, and the resulting supernatant was either used immediately or snap-frozen at $-80^{\circ} \mathrm{C}$ for later use. Homogenates were incubated either at $37^{\circ} \mathrm{C}$ for 30 minutes with $1 \mathrm{mM}$ GTP and $10 \mu \mathrm{M}$ taxol to promote microtubule polymerization or at $4^{\circ} \mathrm{C}$ for 30 minutes to inhibit microtubule polymerization. The resulting reactions were then layered over a $40 \%$ sucrose cushion, centrifuged at $48,000 \mathrm{~g}$ for 30 minutes to pellet the microtubules and microtubule-associated proteins, and processed as above.

For the direct microtubule-binding assay, $1 \mu \mathrm{M}$ of recombinantly expressed and purified 6xHis-tagged CEP290 truncation $\mathrm{M}$ was incubated at $30^{\circ} \mathrm{C}$ for 30 minutes with increasing amounts of pure, prepolymerized microtubules. Reactions were centrifuged and subjected to SDS-PAGE as above, and tubulin and CEP290 truncation $\mathrm{M}$ were detected by Coomassie blue staining.

Bioinformatic analysis. The helical wheel projection shown in Figure 3B was adapted from the Helical Wheel Projection applet available at http:// rzlab.ucr.edu/scripts/wheel/wheel.cgi. The multiple sequence alignment presented in Figure 3C was adapted from GeneDoc 2.7.000.

Statistics. The statistical significance of the difference between 2 means was determined using a 2 -tailed Student's $t$ test. The statistical significance of the difference between 3 or more means was determined using a 2-way ANOVA and Tukey's HSD test. Statistical analysis was performed using GraphPad Prism Software 5.0b. $P<0.05$ was considered significant.

Study approval. The genetics and cell studies were approved by the University of Pennsylvania (protocol 808828, J. Bennett, PI). The animal studies were approved by the University of Pennsylvania IACUC (protocol 802901 , J. Bennett, PI).

\section{Acknowledgments}

We are grateful to Jeannette Bennicelli, George T. Drivas, Michael P. Hart, and Adam Wojno for their many suggestions, conversations, and critical reading of this manuscript. We would also like to thank Jasmine Zhao for her technical support. Plasmids purchased from Addgene were kindly deposited by Dominic Esposito and David Root. Studies in this work were supported by the Wyk Grousbeck Family Foundation and were performed in collaboration with Project CEP290. Additional support was provided by the Foundation Fighting Blindness, Research to Prevent Blindness, NIH R24Ey019861 and 8DP1EY023177, TreatRush (European Union), the Mackall Foundation Trust, the Penn Genome Frontiers Institute, and the F.M. Kirby Foundation. E.L.F. Holzbaur is supported by NIH grant GM48661.

Received for publication June 3, 2013, and accepted in revised form July 25, 2013.

Address correspondence to: Jean Bennett, 310 Stellar-Chance, 422 Curie Boulevard, Philadelphia, Pennsylvania 19104, USA. Phone: 215.898.0915; Fax: 215.573.7155; E-mail: jebennet@mail. med.upenn.edu.
1. Kobayashi T, Dynlacht BD. Regulating the transition from centriole to basal body. J Cell Biol. 2011;193(3):435-444.

2. Davenport JR, et al. Disruption of intraflagellar transport in adult mice leads to obesity and slow-onset cystic kidney disease. Curr Biol. 2007;17(18):1586-1594.

3. Han Y-G, et al. Dual and opposing roles of primary cilia in medulloblastoma development. Nat Med. 2009;15(9):1062-1065.

4. Wong SY, et al. Primary cilia can both mediate and suppress Hedgehog pathway-dependent tumorigenesis. Nat Med. 2009;15(9):1055-1061.

5. Waters AM, Beales PL. Ciliopathies: an expanding disease spectrum. Pediatr Nephrol. 2011;26(7):1039-1056.

6. D'Angelo A, Franco B. The dynamic cilium in human diseases. Pathogenetics. 2009;2(1):3.
7. Perrault I, et al. Spectrum of NPHP6/CEP290 mutations in Leber congenital amaurosis and delineation of the associated phenotype. Hum Mutat. 2007;28(4):416.

8. Den Hollander AI, Roepman R, Koenekoop RK, Cremers FPM. Leber congenital amaurosis: genes, proteins and disease mechanisms. Prog Retin Eye Res. 2008;27(4):391-419.

9. Sayer JA, et al. The centrosomal protein nephrocystin- 6 is mutated in Joubert syndrome and activates transcription factor ATF4. Nat Genet. 2006;38(6):674-681.

10. Valente EM, et al. Mutations in CEP290, which encodes a centrosomal protein, cause pleiotropic forms of Joubert syndrome. Nat Genet. 2006; 38(6):623-625.

11. Brancati F, et al. CEP290 mutations are frequent- ly identified in the oculo-renal form of Joubert syndrome-related disorders. Am J Hum Genet. 2007;81(1):104-113.

12. Helou J, et al. Mutation analysis of NPHP6/CEP290 in patients with Joubert syndrome and Senior-Løken syndrome. J Med Genet. 2007;44(10):657-663.

13. Baala L, et al. Pleiotropic effects of CEP 290 (NPHP6) mutations extend to Meckel syndrome. Am J Hum Genet. 2007;81(1):170-179.

14. Chang B, et al. In-frame deletion in a novel centrosomal/ciliary protein CEP290/NPHP6 perturbs its interaction with RPGR and results in early-onset retinal degeneration in the $\operatorname{rd} 16$ mouse. Hum Mol Genet. 2006;15(11):1847-1857.

15. Tsang WY, et al. CP110 suppresses primary cilia formation through its interaction with CEP290, a protein deficient in human ciliary disease. Dev Cell. 
2008;15(2):187-197.

16. Spektor A, Tsang WY, Khoo D, Dynlacht BD. Cep97 and CP110 suppress a cilia assembly program. Cell. 2007;130(4):678-690.

17. Goetz SC, Liem KF Jr, Anderson KV. The spinocerebellar ataxia-associated gene Tau tubulin kinase 2 controls the initiation of ciliogenesis. Cell. 2012;151(4):847-858

18. Pedersen LB, Rosenbaum JL. Intraflagellar transport (IFT) role in ciliary assembly, resorption and signalling. Curr Top Dev Biol. 2008;85:23-61.

19. Craige B, et al. CEP290 tethers flagellar transition zone microtubules to the membrane and regulates flagellar protein content. J Cell Biol. 2010;190(5):927-940.

20. Kim J, Krishnaswami SR, Gleeson JG. CEP290 interacts with the centriolar satellite component PCM-1 and is required for Rab8 localization to the primary cilium. Hum Mol Genet. 2008;17(23):3796-3805.

21. Stowe TR, Wilkinson CJ, Iqbal A, Stearns T. The centriolar satellite proteins Cep72 and Cep290 interact and are required for recruitment of BBS proteins to the cilium. Mol Biol Cell. 2012;23(17):3322-3335.

22. Williams CL, et al. MKS and NPHP modules cooperate to establish basal body/transition zone membrane associations and ciliary gate function during ciliogenesis. J Cell Biol. 2011;192(6):1023-1041.

23. Hu Q, Nelson WJ. Ciliary diffusion barrier: the gatekeeper for the primary cilium compartment. Cytoskeleton (Hoboken). 2011;68(6):313-324.

24. Kee HL, et al. A size-exclusion permeability barrier and nucleoporins characterize a ciliary pore complex that regulates transport into cilia. Nat Cell Biol. 2012;14(4):431-437.

25. Gorden NT, et al. CC2D2A is mutated in Joubert syndrome and interacts with the ciliopathy-associated basal body protein CEP290. Am J Hum Genet. 2008;83(5):559-571.
26. Schäfer T, et al. Genetic and physical interaction between the NPHP5 and NPHP6 gene products. Hum Mol Genet. 2008;17(23):3655-3662.

27. Moradi P, Davies WL, Mackay DS, Cheetham ME, Moore AT. Focus on molecules: centrosomal protein 290 (CEP290). Exp Eye Res. 2011;92(5):316-317.

28. Coppieters F, Lefever S, Leroy BP, De Baere E. CEP290, a gene with many faces: mutation overview and presentation of CEP290base. Hum Mutat. 2010;31(10):1097-1108.

29. Barral DC, et al. Arl13b regulates endocytic recycling traffic. Proc Natl Acad Sci U S A. 2012; 109(52):21354-21359.

30. Cevik S, et al. Joubert syndrome Arl13b functions at ciliary membranes and stabilizes protein transport in Caenorhabditis elegans. J Cell Biol. 2010;188(6):953-969.

31. Li Y, Wei Q, Zhang Y, Ling K, Hu J. The small GTPases ARL-13 and ARL-3 coordinate intraflagellar transport and ciliogenesis. J Cell Biol. 2010;189(6):1039-1051.

32. Mu FT, et al. EEA1, an early endosome-associated protein. EEA1 is a conserved alpha-helical peripheral membrane protein flanked by cysteine "fingers" and contains a calmodulin-binding IQ motif. J Biol Chem. 1995;270(22):13503-13511.

33. Seaman MN, Sowerby PJ, Robinson MS. Cytosolic and membrane-associated proteins involved in the recruitment of AP-1 adaptors onto the trans-Golgi network. J Biol Chem. 1996;271(41):25446-25451.

34. Klee CB. Ca2+-dependent phospholipid- (and membrane-) binding proteins. Biochemistry. 1988;27(18):6645-6653.

35. Cornell RB, Taneva SG. Amphipathic helices as mediators of the membrane interaction of amphitropic proteins, and as modulators of bilayer physical properties. Curr Protein Pept Sci. 2006;7(6):539-552.

36. Gustke N, Trinczek B, Biernat J, Mandelkow
EM, Mandelkow E. Domains of tau protein and interactions with microtubules. Biochemistry. 1994;33(32):9511-9522.

37. Kikkawa M, Okada Y, Hirokawa N. 15 A resolution model of the monomeric kinesin motor, KIF1A. Cell. 2000;100(2):241-252.

38. Cideciyan $\mathrm{AV}$, et al. Cone photoreceptors are the main targets for gene therapy of NPHP5 (IQCB1) or NPHP6 (CEP290) blindness: generation of an all-cone Nphp6 hypomorph mouse that mimics the human retinal ciliopathy. Hum Mol Genet. 2011;20(7):1411-1423.

39. Zhang Y, et al. BBS mutations modify phenotypic expression of CEP290-related ciliopathies [published online ahead of print August 20,2013]. Hum Mol Genet. doi:10.1093/hmg/ddt394.

40. Weber KL, Sokac AM, Berg JS, Cheney RE, Bement WM. A microtubule-binding myosin required for nuclear anchoring and spindle assembly. Nature. 2004;431(7006):325-329.

41. Moen RJ, Johnsrud DO, Thomas DD, Titus MA. Characterization of a myosin VII MyTH/FERM domain. J Mol Biol. 2011;413(1):17-23.

42. Alieva IB, Gorgidze LA, Komarova YA, Chernobelskaya OA, Vorobjev IA. Experimental model for studying the primary cilia in tissue culture cells. Membr Cell Biol. 1999;12(6):895-905.

43. D'Angiolella V, et al. SCF(Cyclin F) controls centrosome homeostasis and mitotic fidelity through CP110 degradation. Nature. 2010;466(7302):138-142.

44. Smith WJ, Nassar N, Bretscher A, Cerione RA, Karplus PA. Structure of the active N-terminal domain of Ezrin. Conformational and mobility changes identify keystone interactions. J Biol Chem. 2003;278(7):4949-4956.

45. Song X, et al. A novel membrane-dependent on/off switch mechanism of talin FERM domain at sites of cell adhesion. Cell Res. 2012;22(11):1533-1545. 